1 "This is the peer reviewed version of the following article: Top. Curr. Chem. 2017, 375 (15), 1-28, which has been published 2 in final form at DOI: 10.1007/s41061-016-0101-8. This article may be used for non-commercial purposes in accordance 3 with the Terms and Conditions for Self-Archiving published by Springer at https://link.springer.com/." 


\section{Synthesis of Carbonates from Alcohols and $\mathrm{CO}_{2}$}

2

Nicole Kindermann, ${ }^{[a]}$ Tharun Jose, ${ }^{[a]}$ and Arjan W. Kleij ${ }^{[a][b]^{*}}$

4

$5 \quad{ }^{[a]}$ Institute of Chemical Research of Catalonia (ICIQ), the Barcelona Institute of Science and Technology, Av. Països Catalans 16, 43007 - Tarragona (Spain).

Email: akleij@iciq.es

Abstract Alcohols are ubiquitous compounds in nature that offer modular building blocks for synthetic chemistry. Here we discuss the most recent development of different classes of alcohols and their coupling chemistry with carbon dioxide as to afford linear and cyclic carbonates, the challenges associated with their formation and the potential of this chemistry to revive a waste carbon feed stock.

Keywords Carbon Dioxide - Carboxylative Cyclization - Cyclic Carbonates - Diols • 


\section{$\underline{\text { Contents }}$}

2

3

4

5

6

7

8

\section{Synthesis of Acyclic Organic Carbonates}

1.1 The Importance of the Formation of Acyclic Carbonates from Alcohols

1.2 Organic Promoters

1.3 Metal-Based Homogeneous Catalysis

1.4 Metal-based Heterogeneous Catalysis

1.5 Prospects of Acyclic Carbonate Formation

\section{Cyclic Organic Carbonates from Saturated Alcohols}

2.1 Synthesis of Five-membered Cyclic Carbonates

2.1.1 Metal based Catalysts

2.1.2 Organocatalysts

\section{Formation of Six-membered Cyclic Carbonates}

3.1 Metal based catalysts

3.2 Organocatalysts

4. Cyclic Carbonates derived from Unsaturated Alcohols

4.1 Metal based catalysts

4.2 Organocatalysts

\section{Cyclic Carbonates from Halo-Alcohols}

6. Conclusions and Outlook

\section{References}




\section{Synthesis of Acyclic Organic Carbonates}

2

3

4

\subsection{The Importance of the Formation of Acyclic Carbonates from Alcohols}

Among various organic molecules which can in principle be derived from $\mathrm{CO}_{2}$ and alcohols, acyclic carbonates such as dimethyl carbonate (DMC), diethyl carbonate (DEC) and diphenyl carbonate (DPC) have attracted considerable attention over the past decades [1,2]. Especially DMC has been a focus of active recent research in the field, since it represent a multifunctional molecule that can be applied as an apolar solvent, a fuel additive, or as an organic reagent in the production of higher carbonates including polycarbonates, polyurethanes and isocyanates [3-5].

Conventionally, DMC has been produced either from oxidative carbonylation of methanol or through a reaction between methanol and phosgene (Scheme 1) [4]. Both processes cannot be regarded neither sustainable nor environmentally benign, since they use highly toxic and corrosive reagents, and require expensive catalysts in the case of the oxidative carbonylation or disposal of hydrogen chloride when using phosgene. A much more attractive way to produce DMC (and related acyclic carbonates) would be the direct reaction between $\mathrm{CO}_{2}$ and methanol, as shown in Scheme 1. The sole byproduct of this process is water, and its atom economy is comparable to that of the oxidative carbonylation of methanol.

Phosgene route:
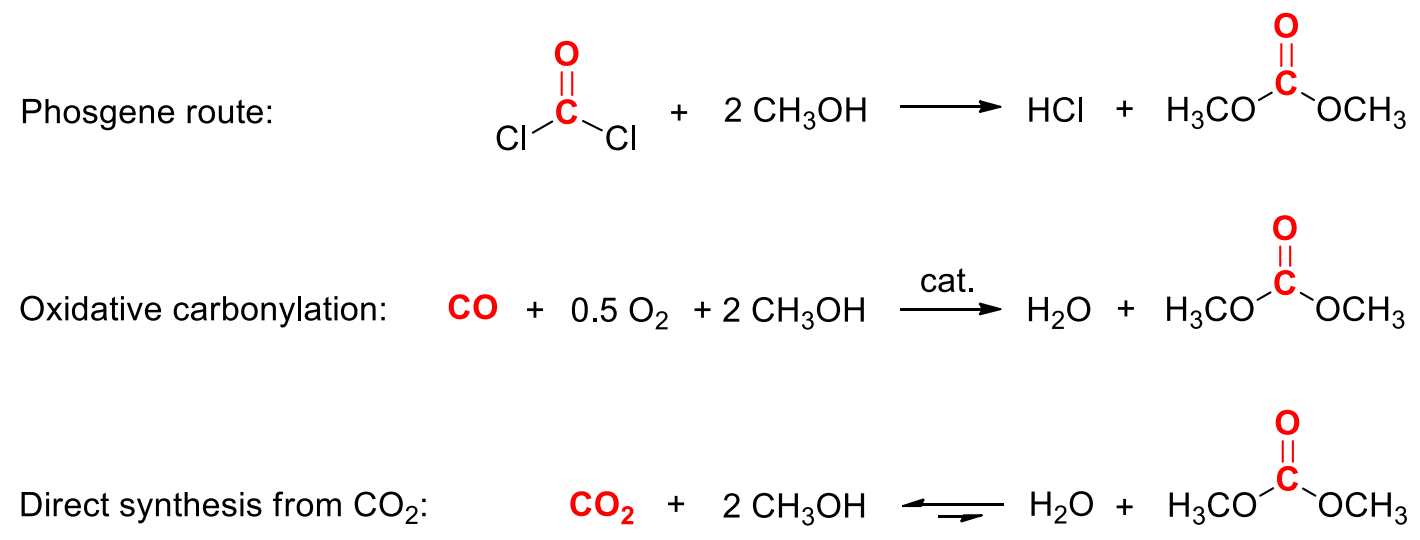

Scheme 1 Different routes for the synthesis of dimethyl carbonate (DMC)

One major drawback of this reaction, however, is its equilibrium limitation (thermodynamics) providing only (very) low yields in DMC. High pressures of $\mathrm{CO}_{2}$ might help to partially overcome these limitations [6] but at the cost of a high energy demand accompanying this pressurization. Removal of the water formed in the DMC synthesis is 
another way to shift the equilibrium towards the desired product DMC. Accordingly, the development of efficient catalysts and in combination with a dehydrating agent has become one major focus in the synthesis of acyclic carbonates [7]. Besides DMC, also DEC and DPC have relevance in industrial processes [8, 9]. DPC has already found wide application in polycarbonate synthesis being efficiently used for transesterification of Bisphenol A (BPA). The derived polycarbonate is a thermoplastic polymer used on a large scale as a material for numerous applications (including electrical insulation) with a production of more than a billion tons per year; apart from the DPC route, the major process towards BPA based polycarbonates still relies on the direct reaction of BPA with phosgene. DPC synthesis from phosgene or through oxidative carbonylation, comparable to DMC synthesis, has technical and logistic disadvantages. Transesterification of DMC with phenol is an attractive alternative to the conventional synthesis methods, however, in order to make the overall process "greener", DMC needs to be produced in a sustainable way.

For the direct synthesis of DMC from methanol and $\mathrm{CO}_{2}$, efficient removal of water - beside the adequate choice of a catalyst - seems to be crucial in order to achieve an improvement of this promising methodology and to increase its relevance in industrial synthesis. Thus, the following sections will concentrate on organic and inorganic promoters for DMC formation, and the importance of dehydrating agents.

\subsection{Organic Promoters}

One approach to address the equilibrium limitation is based on organic molecules acting as promoters of DMC formation. Activation of $\mathrm{CO}_{2}$ or water capture might both be performed by the same organic molecule [10-12]. Especially in the latter scenario, a stoichiometric use of the promoter is required, since water is usually bound irreversibly. For instance, Aresta and coworkers established the use of dicyclohexylcarbodiimide (DCC; Scheme 2) for the direct synthesis of DMC from $\mathrm{CO}_{2}$ and methanol under mild conditions (e.g. $80^{\circ} \mathrm{C}$ and $5.0 \mathrm{MPa}$ of $\mathrm{CO}_{2}$ ). Based on the use of DCC, they reported yields of up to $62 \%$ for DMC after only $6 \mathrm{~h}$, but the protocol could also be successfully employed in the conversion of ethanol or allyl alcohol substrates thereby giving access to other dialkyl carbonates. Mechanistic and computational studies led to a putative mechanism, with an isourea/hemi-carbonate adduct as the proposed key intermediate (Scheme 2). 


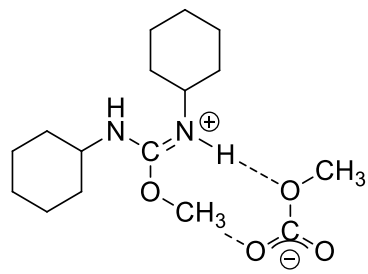

$\mathrm{Ph}_{3} \mathrm{P} /$ EtOOC-N=N-COOEt

Mitsunobu's reagent

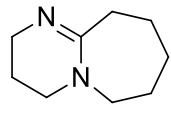

DBU

Scheme 2 Organic promotors for the direct formation of DMC from alcohols and $\mathrm{CO}_{2}$

A different synthetic route towards DMC synthesis, which gives access to symmetric and asymmetric acyclic carbonates, is based on Mitsunobu's reagent. This methodology, developed by Chaturvedi et al. [11] can furthermore be successfully employed to convert primary, secondary and even tertiary alcohols in a one-pot reaction with good to high yields (70-98\%) in all reported cases. Recently, the successful application of DBU for the synthesis of acyclic carbonates was demonstrated by Jang and coworkers [12]. The scope comprised the synthesis of various cyclic and acyclic organic carbonates, including DMC, which could be obtained in $48 \%$ yield under comparatively mild reaction conditions $\left(70^{\circ} \mathrm{C}, 10\right.$ bar $\left.\mathrm{CO}_{2}\right)$.

\subsection{Metal-Based Homogeneous Catalysis}

Among the homogeneous metal catalysts considered, metal alkoxides have been intensively studied in DMC synthesis since they have been shown to absorb $\mathrm{CO}_{2}$ to form organic carbonates $[13,14]$. Besides titanium, zirconium and niobium compounds [14-18], tin(IV) complexes have been investigated in detail with respect to DMC formation from methanol and carbon dioxide. Tetraalkoxy $\left[\mathrm{Sn}(\mathrm{OR})_{4}\right]$ as well as dialkoxydialkyl $\left[\mathrm{R}_{2}^{1} \mathrm{Sn}\left(\mathrm{OR}^{2}\right)_{2}\right]$ tin compounds are classes of organometallics both active in DMC formation, even though the efficiency of the reported systems remains rather low [18-20]. Significant improvements of the methodology were made by Sakakura et al. by investigating the effect of different drying agents on the DMC formation catalyzed by organometallic tin compounds. Initially starting with orthoesters, DMC yields of $48 \%$ (based on the orthoester reagent) and a selectivity of $85 \%$ (DMC) were found under high pressures of $\mathrm{CO}_{2}(300 \mathrm{~atm})$ and reaction temperatures of $180^{\circ} \mathrm{C}$, using $\left[\mathrm{Bu}_{2} \mathrm{Sn}(\mathrm{OMe})_{2}\right]$ as catalyst [21]. Notably, a substantial enhancement of the catalyst performance by the addition of onium salts was observed. One drawback of this approach, however, was the required 
stoichiometric use of orthoesters. As shown in Scheme 3, they can capture water under the release of an alcohol and an ester, but recycling of the desiccant is not feasible.

Orthoester:

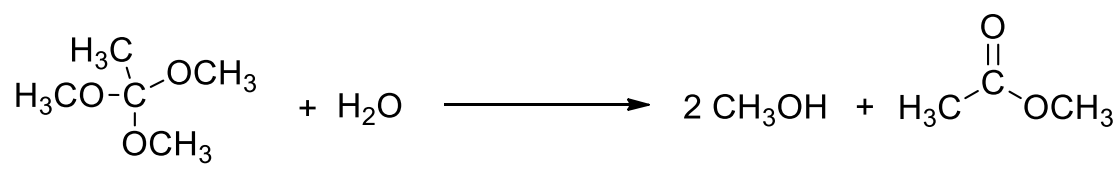

Acetal:

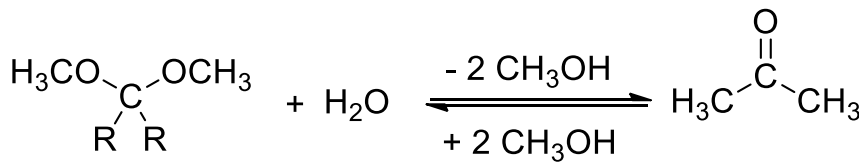

\section{Scheme 3 Organic desiccants that have been employed as water-capturing agents}

By contrast, drying agents such as acetals promised to be more sustainable, since they feature recovery potential from the formed ketone that upon reaction with alcohols can regenerate the acetal [22]. In comparison to the orthoester system, a combination of tin compound and acetal performed slightly better with a DMC yield of $58 \%$ based on the acetal. Even though in this case onium salts do not lead to improved catalyst performance, more recently it has been shown that acidic co-catalysts have a pronounced influence on the efficiency in DMC formation, and for instance the presence of small amounts of co-catalytic $\mathrm{Ph}_{2} \mathrm{NH}_{2} \mathrm{OTf}$ accelerated the reaction substantially [15].

Besides organic desiccants, also inorganic versions such as molecular sieves have been successfully employed in the dehydration process [6]. Even though zeolites are not considered to be very efficient under high reaction temperature conditions, yields up to $45 \%$ based on $\mathrm{MeOH}$ were reported [6]. Mechanistic proposals are based on early structural findings [23], as depicted in Scheme 4. After $\mathrm{CO}_{2}$ insertion into the metal methoxide moiety, the bridging alkoxide reacts with the hemi-carbonate anion with subsequent DMC release. The active species can be reestablished from the corresponding oxide or hydroxide by reacting with methanol under the release of water [24]. Recent reinvestigations and density functional theory (DFT) calculation, though, suggest that the actual active intermediates might be stannoxane dimers (Scheme 4) [25, 26]. 

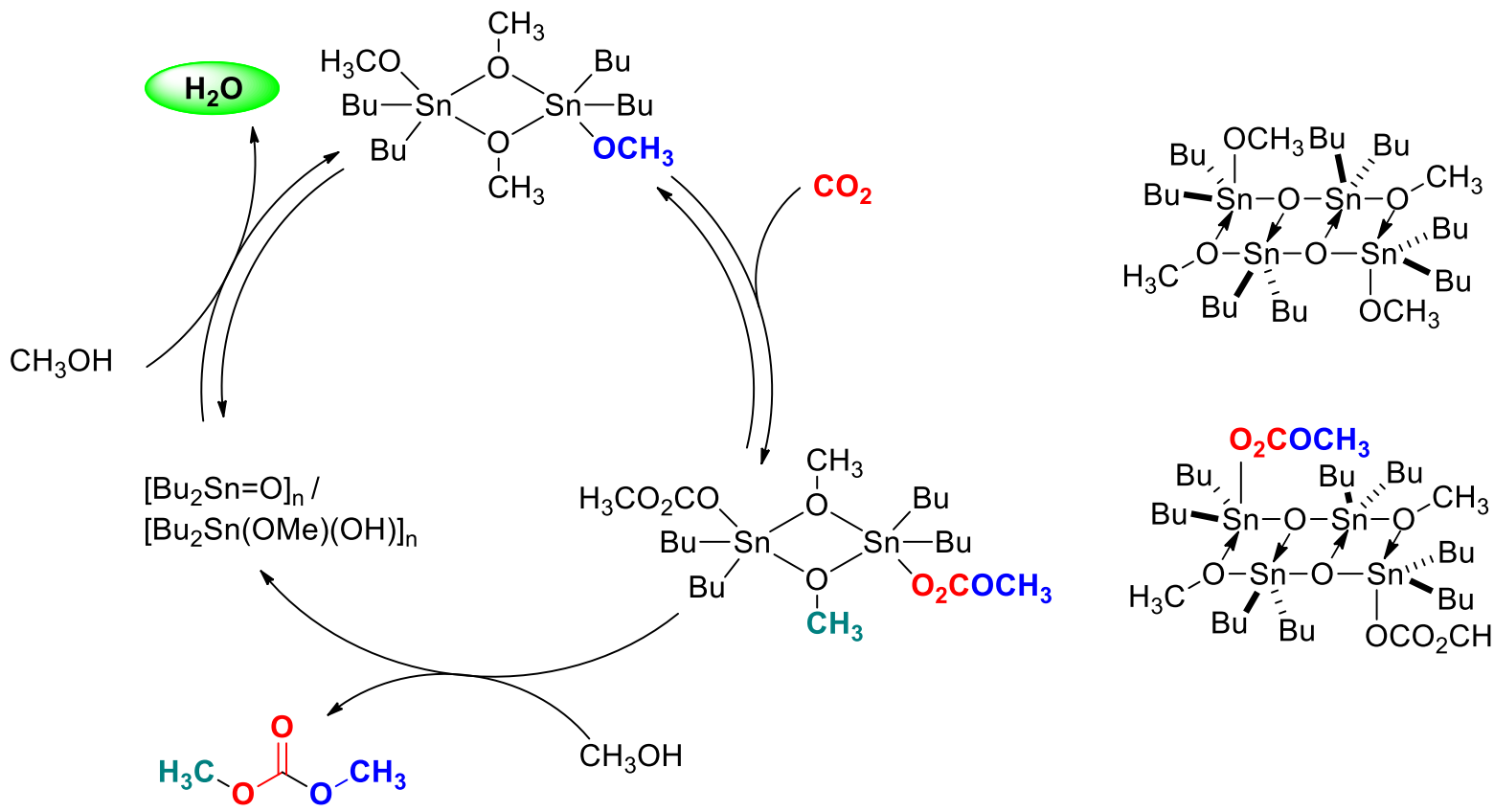

Scheme 4 Mechanistic proposal based on structural findings (left); reactive intermediates suggested by computational and experimental studies

\subsection{Metal-based Heterogeneous Catalysis}

6

The use of heterogeneous catalysts in the synthesis of chemical compounds has several key benefits if compared with homogenous catalysis. Separation of the catalyst from the products is usually straightforward, e.g. by a simple filtration. At the same time, the ease of separation is advantageous when it comes to recyclability of the catalyst. This makes heterogeneous catalysts an interesting choice for industry, especially when similar selectivities and activities as in the case of homogenous catalysts can be achieved. For DMC synthesis, the use of metal oxides had a considerable impact on the field. Besides main group metal oxides such as $\mathrm{Mg}-\mathrm{Al}$ hydrotalcites [27, 28], mainly transition metal oxides have been employed. Among these are vanadium oxides, doped with Brønsted acids [29] or copper/nickel [30], but the most widely studied systems consist of zirconium and cerium oxides.

Early work by Tomishige and Fujimoto revealed the great potential of the amphoteric materials $\mathrm{ZrO}_{2}$ and $\mathrm{CeO}_{2}$ (or solid solution mixtures) [31-35], being mainly attributed to synergistic effects between their acidic and basic sites [31]. However, for the simple metal oxides the equilibrium restriction did not allow for yields exceeding 2\% [34] even under high $\mathrm{CO}_{2}$ pressures of $6 \mathrm{MPa}$ and temperatures around $127^{\circ} \mathrm{C}$. Doping of the metal oxides with 
1 Brønsted acidic sites using $\mathrm{H}_{3} \mathrm{PO}_{4}$ or $\mathrm{H}_{3} \mathrm{~W}_{12} \mathrm{O}_{40}$ led to slightly improved yields or shorter reaction times under comparable conditions [36-38].

Major breakthroughs were only achieved, though, if dehydrating agents were added. In contrast to orthoesters or acetals, which were formerly used by Sakakura, Tomishige and coworker suggested the use of nitriles in 2009 [39, 40]. Water capture with nitriles leads to amides, which can later be converted back to the corresponding nitrile and enable the regeneration of the dehydrating species. The elegance of this synthetic route is based on the simultaneous conversion of $\mathrm{CO}_{2}$ and methanol to DMC and the nitrile hydration to the corresponding amide by $\mathrm{CeO}_{2}$. If using acetonitrile as desiccant at $0.5 \mathrm{MPa} \mathrm{CO}_{2}$ pressure and $150{ }^{\circ} \mathrm{C}$, the yield of DMC after $48 \mathrm{~h}$ reached about $9 \%$ but with only a mediocre selectivity for DMC of $65 \%$. Also the selectivity of amide formation upon water capture remained an issue, especially in the light of recyclability of the dehydrating agent. Benzonitrile proved to be a much better choice not only with respect to selective amide formation, but its use also increased the DMC yield to a remarkable $47 \%\left(1 \mathrm{MPa}, 150{ }^{\circ} \mathrm{C}, 86 \mathrm{~h}\right)$, with a significantly improved chemo-selectivity of $75 \%$ [41].

As suggested by the authors, the reason for this improved reactivity/selectivity behavior might be suppression of competitive alcoholysis of the formed amide, if compared to acetamide that is in situ produced from acetonitile. A systematic screening for suitable nitrile-based dehydrating agents $[42,43]$, which are efficiently hydrated by $\mathrm{CeO}_{2}$, finally led to the use of 2cyanopyridine as the preferred nitrile in combination with a cerium oxide catalyst. With this system (5 MPa, $120{ }^{\circ} \mathrm{C}, 12 \mathrm{~h}$ ), yield of and selectivity for DMC were extraordinarily high, reaching levels of $94 \%$ and $96 \%$, respectively. The recycling of the formed amide was also addressed, and the dehydration of 2-picolinamide by $\mathrm{Na}_{2} \mathrm{O} / \mathrm{SiO}_{2}$ was shown to be feasible even though the overall efficiency should be improved. The scope is not only limited to the formation of DMC, but also ethanol or branched alcohols could be converted in a similar way albeit with a drop in yield of the corresponding carbonate product.

In order to provide a lead for further improvement of the catalytic system, mechanistic insights are inevitable. Tomishige et al. proposed a reaction cycle based on kinetic, spectroscopic and computational studies, leading to an overall mechanism as depicted in Scheme 5 [44]. It resembles the mechanism suggested by Bell et al. for the zirconium oxide catalyzed formation of $\mathrm{DMC}$ from methanol and $\mathrm{CO}_{2}$ [45]. A molecule of $\mathrm{CO}_{2}$ inserts into the $\mathrm{Ce}-\mathrm{OCH}_{3}$ bond of surface bound methanol to yield a Ce-methyl carbonate species. For the next step the authors suggested a nucleophilic attack of another surface bound methoxy group 
to give DMC. The formed hydroxide species on the cerium oxide surface can subsequently

2 react with 2-cyanopyridine and result in the formation of 2-picolinamide [46, 47]. Other

3 authors, however, claim the attack of gas-phase methanol [48], or the formation of a

4 carbomethoxide intermediate on the cerium oxide surface [49]. Therefore, the exact mechanism

5 concerning the cerium oxide mediated formation of DMC remains subject of ongoing debate in

6 the literature.

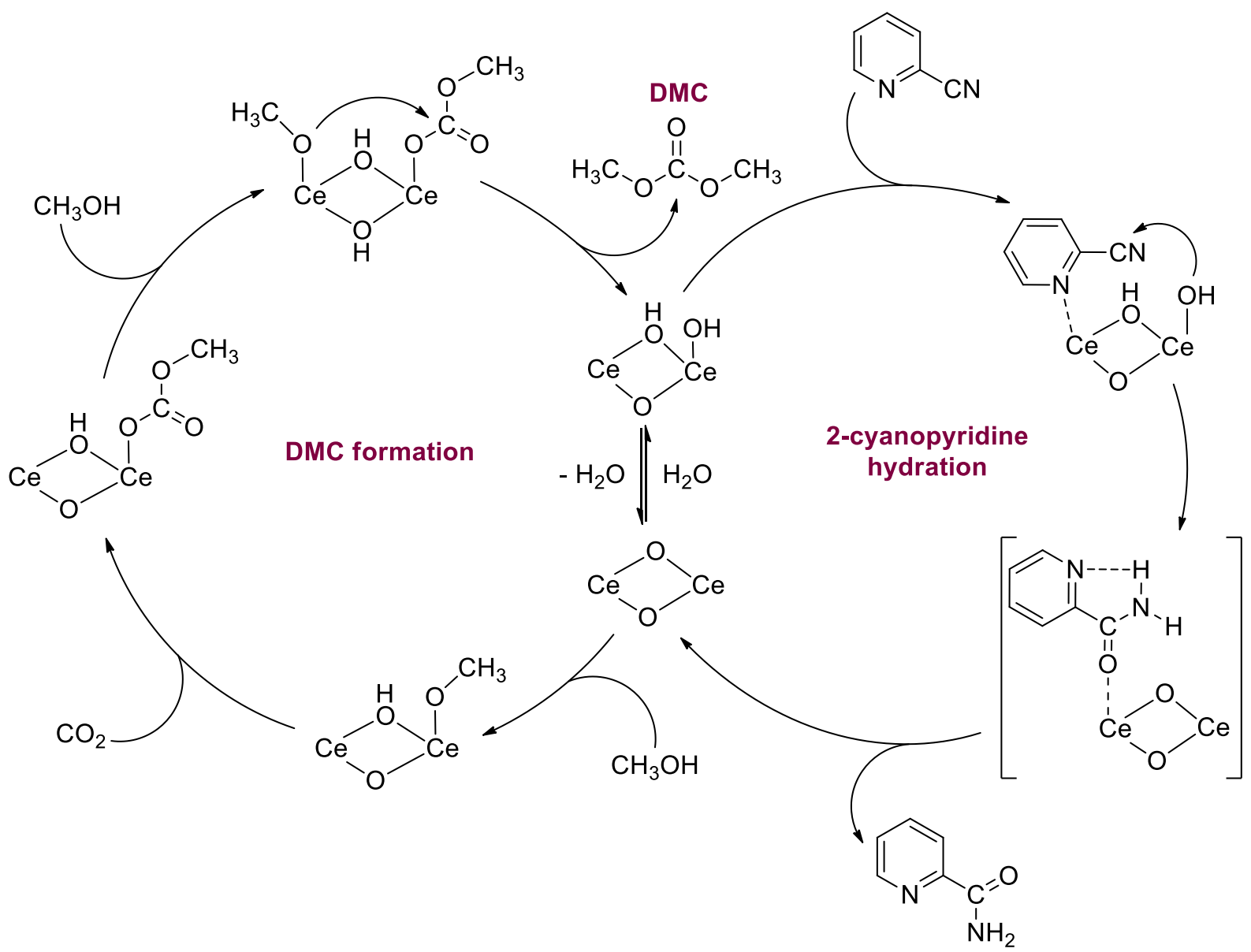

Scheme 5 Mechanism of cerium oxide catalyzed simultaneous formation of DMC and 2picolinamide formation from $\mathrm{MeOH} / \mathrm{CO}_{2}$ and 2-cyanopyridine, respectively

Its superb performance regarding yield and selectivity towards DMC formation makes the combination of cerium(IV) oxide and 2-cyanopyridine a promising candidate for further commercial applications. This is reflected in the development of the first continuous flow process using fixed bed reactors [50], as well as intensified research concerning the reusability of the cerium oxide catalyst, which eventually suffers from deactivation by adsorption of the formed amide [44, 51]. Interestingly, the scope of this system is not limited to the formation of simple acyclic carbonates, but can also be employed to cyclic carbonates (see section 2 and 3 
of this review) [52], as well as cyclic and acyclic carbamates and urea derivatives [53-55]. Very recently, cerium oxide and 2-cyanopyridine was furthermore used to synthesize polymeric materials from $\mathrm{CO}_{2}$ and diols, as depicted in Scheme 6 [56]. Despite the low molecular weight of the produced materials, this represents the first exciting example of a direct copolymerisation between $\mathrm{CO}_{2}$ and diols.

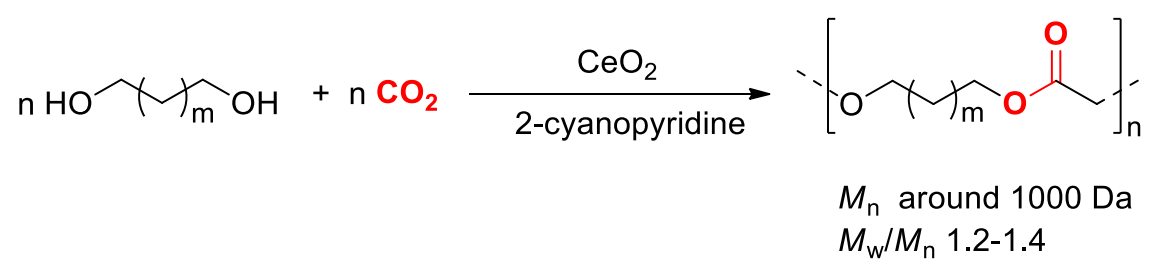

Scheme 6 Direct polycarbonate formation from diols and carbon dioxide

\subsection{Prospects of Acyclic Carbonate Formation}

Over the last two decades, huge progress has been made regarding the yield and chemoselectivity in the production of DMC from methanol and carbon dioxide. Homogeneous and heterogeneous catalyst systems have been improved continuously, going hand in hand with the development of efficient dehydrating systems to shift the reaction equilibrium towards the formation of DMC. Various studies were dedicated to homogeneous metal alkoxide compounds, with tin(IV) complexes leading the way. In combination with different waterremoving agents, good yields and selectivities for the formation of acyclic carbonates were obtained. Even better performances were achieved using heterogeneous, amphoteric metal oxides such as zirconium oxide and cerium oxide, with the latter giving excellent yields and selectivities towards DMC when combined with 2-cyanopyridine as desiccant. Although product separation and recyclability of catalyst and dehydrating agent have been clearly demonstrated, recovery of the nitrile from 2-picolinamide remains problematic for further applications and is at the same time crucial to optimize the cost-benefit ratio as well as to design a truly sustainable, atom-economical route for the preparation of acyclic organic carbonates. New reactor technologies such as the use of flow chemistry with a clever use of recycle streams may at some point in time allow to efficiently run DMC and related synthesis in continuous mode at low conversion of the alcohol without the need for a dehydrating agent. 


\section{Cyclic Organic Carbonates from Saturated Alcohols}

\subsection{Synthesis of Five-membered Cyclic Carbonates}

\subsubsection{Metal based catalysts}

From an industrial point of view, the transformation of ethylene glycol (EG) and propylene glycol (PG) is of high interest. Both EG and PG are byproducts obtained in the transesterification of ethylene carbonate (EC) and propylene carbonate (PC), respectively, using $\mathrm{MeOH}$ as a reagent and with the aim to produce DMC. Thus, recycling of the EG or PG by reaction with $\mathrm{CO}_{2}$ has the potential to lift the overall sustainability of DMC synthesis from EC or PC (Scheme 7) [57].

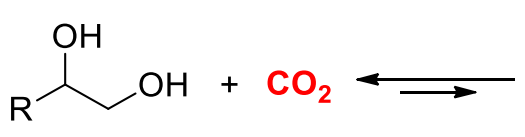

EG: $R=H$

PG: $\mathrm{R}=\mathrm{Me}$

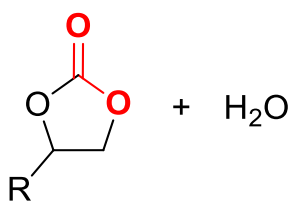

$E C: R=H$

PC: $\mathrm{R}=\mathrm{Me}$

Scheme 7 Synthesis of cyclic carbonates from $\mathrm{CO}_{2}$ and the glycols EG and PG

Tomishige reported the first attempt for this transformation using a calcined $\mathrm{CeO}_{2}-\mathrm{ZrO}_{2}$ catalyst system in the presence of acetonitrile [58, 59]. Conversions of 0.7 and $1.6 \%$ were obtained under optimal reaction conditions using $0.36 \mathrm{mmol}$ (total amount of $\mathrm{Ce}$ and $\mathrm{Zr}$ ) of catalyst weight, $200 \mathrm{mmol}$ of $\mathrm{CO}_{2}, 120 \mathrm{mmol}$ acetonitrile, a reaction temperature of $150{ }^{\circ} \mathrm{C}$ for 2 h: these conditions led to the synthesis of EC and PC from EG and PG, respectively. Interestingly, the EC and PC amount increased when the calcination temperature of the catalyst system was increased, which resulted in lowering the amount of acid/base sites and the surface area. As in the formation of linear carbonates, the reaction mediated by the $\mathrm{CeO}_{2}-\mathrm{ZrO}_{2}$ catalyst system is thermodynamically limited with a maximum conversion of $2 \%$; removal of $\mathrm{H}_{2} \mathrm{O}$ would be crucial to maximize the conversion/yield of the carbonate product.

Subsequently, He et al. reported two different catalyst systems such as dibutyltin oxide/dibutyltin dimethoxide and low toxic magnesium and its oxide for the synthesis of PC from PG and $\mathrm{CO}_{2}$ under supercritical conditions using $N, N$,-dimethylformamide (DMF) as solvent $[60,61]$. In both cases, the maximum conversion obtained for PG was $<4 \%$ with $100 \%$ chemo-selectivity. Also, alkali carbonates were used to catalyze the PC synthesis from PG and 
$1 \mathrm{CO}_{2}$ with a relative high yield of $10.5 \%$ under supercritical conditions in the presence of ammonium carbonate and acetonitrile [62]. Acetonitrile did not only act as a solvent here but also as the dehydrating agent to eliminate the water produced during the reaction thereby shifting the equilibrium towards the formation of PC. However, the hydrolysis of acetonitrile may generate acetamide and can subsequently react with water to form acetic acid and ammonia. Acetic acid can react with PG to produce propylene glycol-2-acetate, lowering the overall selectivity towards PC. Therefore, introduction of ammonium carbonate into the reaction system inhibited the hydrolysis of acetamide and improved the chemo-selectivity toward PC.

The combination of its bio-based origin and wide reactivity has made glycerol carbonate (GC) a versatile and renewable building block for organic chemistry. The direct carboxylation of glycerol (Gly) and $\mathrm{CO}_{2}$ is a very interesting though challenging route that would convert two waste materials from the chemical industry into a valuable product (Scheme 8). Mouloungui et al. attempted to prepare GC under supercritical conditions but the reaction did not occur [63]. Later on, Dibenedetto employed tin-based catalyst systems $\left[n-\mathrm{Bu}_{2} \mathrm{Sn}(\mathrm{OMe})_{2}\right]$ and $\left[n-\mathrm{Bu}_{2} \mathrm{SnO}\right]$ under solvent-free conditions using $6 \mathrm{~mol} \%$ of catalyst $\left[n-\mathrm{Bu}_{2} \mathrm{Sn}(\mathrm{OMe})_{2}\right], 5 \mathrm{MPa}$ of $\mathrm{CO}_{2}$ pressure, a reaction temperature of $180{ }^{\circ} \mathrm{C}$ for $15 \mathrm{~h}$ to obtain a maximum conversion of $6.7 \%$ [64]. Molecular sieves were introduced into the reactor to reduce the water content in the homogeneous phase to favor the equilibrium towards the formation of GC.

Hereafter, Dibenedetto applied mixed oxide catalysts $\left(\mathrm{CeO}_{2} / \mathrm{Al}_{2} \mathrm{O}_{3}\right.$ and $\left.\mathrm{CeO}_{2} / \mathrm{Nb}_{2} \mathrm{O}_{5}\right)$ under the same reaction condition mentioned above in a biphasic system using tetra(ethylene glycol) dimethyl ether (TEGDME) as solvent to obtain a maximum Gly conversion of $2.5 \%$. The catalyst was recyclable at least 3 times without any observable loss of activity [65]. Munshi et al. showed that addition of methanol to the Dibendetto's $n$ - $\mathrm{Bu}_{2} \mathrm{SnO}$-based catalyst system enhanced the catalytic activity and as a result $30 \%$ yield for $\mathbf{G C}$ in $4 \mathrm{~h}$ at $80{ }^{\circ} \mathrm{C}$ and $3.5 \mathrm{MPa}$ $\mathrm{CO}_{2}$ pressure using $1 \mathrm{~mol} \%$ of catalyst could be attained [66]. The reaction with diglyme, an 
inert aprotic solvent, instead of methanol did not improve the yield of $\mathbf{G C}$ and also the use of other alcohols such as ethanol, propanol and butanol slowed down the reaction rate. These combined results indicated that the role of methanol is not just acting as a solvent but it is likely also chemically involved. The proposed mechanism presumes the activation of $n-\mathrm{Bu}_{2} \mathrm{SnO}$ by methanol to give $n-\mathrm{Bu}_{2} \mathrm{Sn}(\mathrm{OMe})_{2}$ which in turn reacts with Gly forming $n$ - $\mathrm{Bu}_{2} \mathrm{Sn}($ glycerol- $2 \mathrm{H})$ and undergoes $\mathrm{CO}_{2}$ insertion, leading eventually to GC via a $\mathrm{Sn}$ (glycerolcarbonate) complex. The catalytic cycle is completed by ligand exchange in the presence of methanol followed by ring-closing, release of the GC product and the reformation of the $\mathrm{Sn}(\mathrm{OMe})_{2}$ complex species as shown in Scheme 9. During the process, the monomeric species $\left(n-\mathrm{Bu}_{2} \mathrm{Sn}(\mathrm{glycerol}-2 \mathrm{H})\right)$ can either incorporate $\mathrm{CO}_{2}$ or oligomerize causing catalyst deactivation. The addition of methanol prevents the formation of an oligomeric species as it is actively involved in the formation of GC whereas the water formed during reaction is removed continuously from the system.

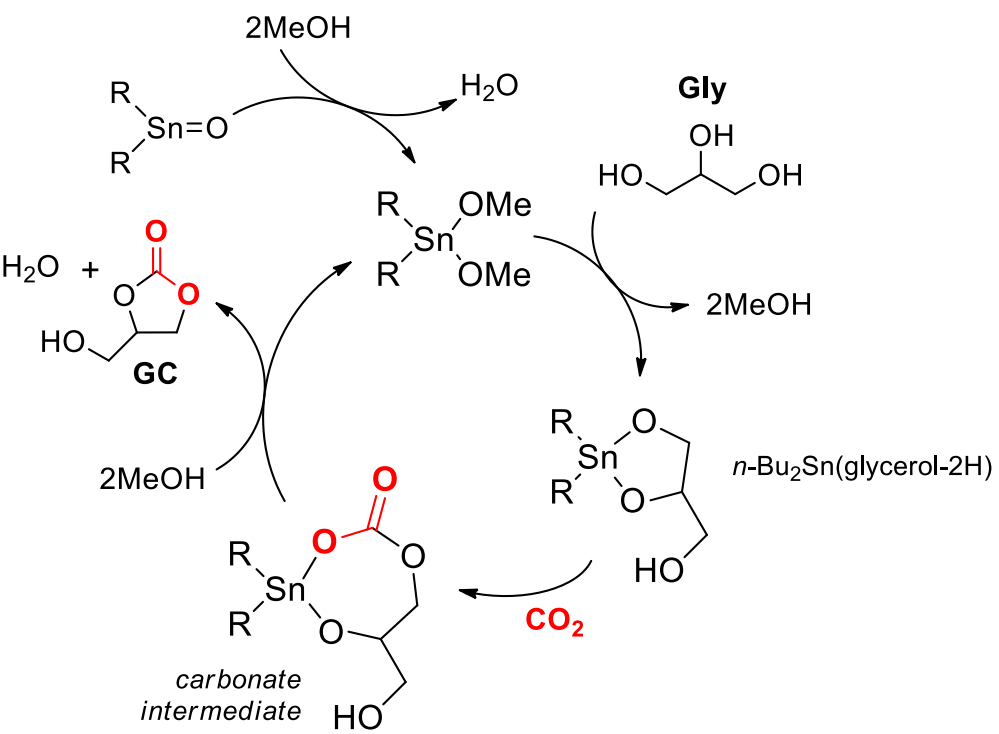

Scheme 9 Proposed reaction path towards GC formation [66]

Similar to Tomishige's work [58] using acetonitrile as a medium that helps to overcome the thermodynamic limitation of the process, Sun and coworkers used acetonitrile as a sacrificial coupling in the presence of a $\mathrm{La}_{2} \mathrm{O}_{2} \mathrm{CO}_{3}-\mathrm{ZnO}$ catalyst system for the transformation of $\mathrm{CO}_{2}$ and Gly into GC [67]. The highest Gly conversion (30.3\%) with a GC yield of $14.3 \%$ was reported at $4 \mathrm{MPa}$ of $\mathrm{CO}_{2}$ pressure, a reaction temperature of $170{ }^{\circ} \mathrm{C}$ after $12 \mathrm{~h}$ when the catalyst system was calcined at $500{ }^{\circ} \mathrm{C}$. The envisioned mechanism involves the activation of Gly by Lewis acidic sites $\left(\mathrm{Zn}^{2+}\right)$ forming a glyceroxide anion, and subsequently the oxygen atom of the adjacent hydroxyl group attacks the zinc cation resulting in zinc-glycerolate species along 
with the formation of a molecule of water which is converted to an amide reacting with acetonitrile.

The uncalcined catalyst produces only a low yield of GC due to the higher content of crystal lattice water, which favors the hydrolysis of acetonitrile ( $c f$., amide formation) and results in more byproducts. With the introduction of $\mathrm{La}_{2} \mathrm{O}_{2} \mathrm{CO}_{3}$, the amount of lattice oxygens ( $\mathrm{La}-\mathrm{O}$ pairs) increases on the surface and leads to an increase of moderately basic sites, which in turn enhance the activation of glycerol and $\mathrm{CO}_{2}$ and thereby exhibit higher catalytic activity than pure $\mathrm{ZnO}$. Likewise, various other catalysts systems such as $\mathrm{Cu} / \mathrm{La}_{2} \mathrm{O}_{3}$ and $\mathrm{Cu}$-supported catalysts, $\mathrm{Zn} / \mathrm{Al} / \mathrm{La}$ and $\mathrm{Zn} / \mathrm{Al} / \mathrm{La} / \mathrm{M}(\mathrm{M}=\mathrm{Li}, \mathrm{Mg}$ and $\mathrm{Zr}$ ) hydrotalcites, and $\mathrm{Zn} / \mathrm{Al} / \mathrm{La} / \mathrm{X}(\mathrm{X}=$ $\mathrm{F}, \mathrm{Cl}, \mathrm{Br}$ ) catalysts were also successfully employed for the direct carbonylation of Gly to obtain maximum conversions of the substrate of $<36 \%$ under typically harsh, supercritical reaction conditions in the presence of acetonitrile [68-70].

Very recently, $\mathrm{He}$ and co-workers obtained $\mathbf{G C}$ from the carbonylation of Gly and $\mathrm{CO}_{2}$ over $\mathrm{CeO}_{2}$ catalysts with the hydrolysis of 2-cyanopyridine as the sacrificial dehydrating agent [71]. Calcined $\mathrm{CeO}_{2}$ with three different morphologies pertinent to nanoparticles, nano-rods and sponge-like nanomaterials were prepared corresponding to three different types of methods being precipitation, hydrothermal treatment and sol-gel methodology, respectively. All three samples showed excellent catalytic performance obtaining GC yields between 20-34 \% under relatively mild reaction conditions $\left(150{ }^{\circ} \mathrm{C}, 4 \mathrm{MPa}, 5 \mathrm{~h}\right)$ with an activity order of nanorod catalyst > catalytic nanoparticles > sponge-like catalyst. The $\mathrm{CeO}_{2}$ nano-rod type catalyst with the most abundant basic sites and oxygen vacancies gave the highest yield of GC, and spongelike $\mathrm{CeO}_{2}$ with medium amount of basic sites and the least defects gave the lowest, indicating that the oxygen vacancies play an important role in the catalytic system. Among various dehydrating agents used, 2-cyanopyridine showed the best performance ascribed to the relatively strong alkalinity and the formation of intramolecular hydrogen bonding in the produced amide when the nitrile reacts with water. By optimizing the reaction conditions, the GC yield could be increased to as high as $78.9 \%$ and the used catalyst could be easily regenerated through the calcination process at $400{ }^{\circ} \mathrm{C}$ for $5 \mathrm{~h}$, and was recycled five times successfully. 


\subsubsection{Organocatalysts}

Jang and co-workers introduced a metal-free carbonylation reaction in which alcohols are converted into corresponding cyclic carbonates in the presence of an organic base and dibromomethane $\left(\mathrm{CH}_{2} \mathrm{Br}_{2}\right)$ as the solvent [11]. The organic base, 1,8diazabicyclo[5.4.0]undec-7-ene (DBU), is assumed to deprotonate the $\mathrm{OH}$ unit of ethylene glycol (EG), which would render it more nucleophilic. DBU is also known to form an adduct with $\mathrm{CO}_{2}$, and can in this way increase the nucleophilic character of $\mathrm{CO}_{2}$ favoring reaction with EG. In the presence of 2 equivalents of $\mathrm{DBU}$ at $0.5 \mathrm{M}$ of $\mathrm{CH}_{2} \mathrm{Br}_{2}$ and at $0.5 \mathrm{MPa} / 70{ }^{\circ} \mathrm{C}, \mathbf{E G}$ $(0.5 \mathrm{mmol})$ was converted into EC (24\% after $18 \mathrm{~h})$. In the presence of an ionic liquid (IL: bmimPF 6 ), the $\mathbf{E C}$-yield was enhanced to $54 \%$ and under higher $\mathrm{CO}_{2}$ pressure $(1 \mathrm{MPa})$ it was further improved to $74 \%$.

ILs are well-known to increase the solubility of $\mathrm{CO}_{2}$ in the reaction media. Under the optimized reaction conditions, various other alcohols were also tested as substrates and it was found that Gly underwent good conversion obtaining a GC yield of $86 \%$. Similarly, other methyl- and phenyl-substituted ethylene diols underwent smooth conversion to their cyclic carbonates to afford yields of 67-79\%. A cyclic diol was also tested and displayed an excellent yield of $73 \%$. A set of additional experiments was performed to understand the operating mechanism in these diol/ $\mathrm{CO}_{2}$ coupling reactions. First, an ${ }^{18} \mathrm{O}$-labeling experiment was conducted with mono- ${ }^{18} \mathrm{O}$-labeled styrene glycol and a $70 \%$ yield of the styrene carbonate was achieved, where the ${ }^{18} \mathrm{O}$ label did not exchanged with ${ }^{16} \mathrm{O}$ during the reaction, i.e. the cyclic carbonate had the ${ }^{18} \mathrm{O}$-labeled atom incorporated. Second, the use of optically active $(S)$-styrene glycol was examined, and the resultant styrene carbonate was analyzed by HPLC showing full retention of the initial configuration.

The mechanism of the aforementioned reaction is shown in Scheme 10 in which the primary alcohol first attacks the DBU- $\mathrm{CO}_{2}$ adduct to form the carbonate intermediate $\mathbf{I}$, which then reacts with $\mathrm{CH}_{2} \mathrm{Br}_{2}$ to form reactive carbonate II. Then the intermolecular attack of the (preactivated) secondary alcohol unit onto the carbonate followed by the elimination of $\mathrm{HOCH}_{2} \mathrm{Br}$ affords the styrene carbonate product. The intramolecular addition of the carbonate nucleophile in intermediate III likely is not competitive under these conditions as supported by the experimental result obtained using $(S)$-styrene glycol. 


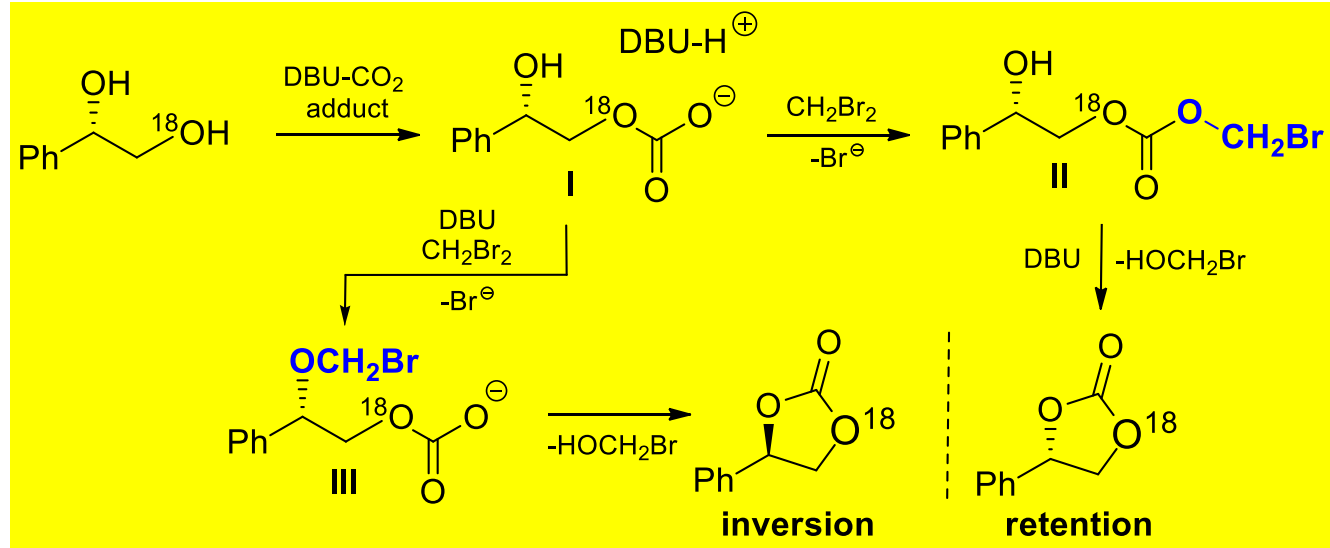

\section{Formation of Six-membered Cyclic Carbonates}

\subsection{Metal based catalysts}

The pioneering group of Tomishige has successfully performed various carboxylation reactions of diols and $\mathrm{CO}_{2}$ to afford their cyclic carbonates. Recently, they employed the privileged $\mathrm{CeO}_{2}$ catalyst in combination with 2-cyanopyridine as dehydrating agent to yield various five- and six-membered cyclic carbonates from $\mathrm{CO}_{2}$ and diols [52]. From a series of different metal oxides combined with 2-cyanopyridine for the synthesis of $\mathbf{P C}$ from $\mathbf{P G}$ and $\mathrm{CO}_{2}, \mathrm{CeO}_{2}$ was shown to be more active by 2 orders of magnitude compared with other metal oxides. 2Cyanopyridine was preselected as nitrile for its exceptional reactivity towards hydration forming 2-picolinamide. Without the addition of 2-cyanopyridine, the PC yield was as low as $0.3 \%$ due to the unfavorable thermodynamics. Addition of $100 \mathrm{mmol}$ of 2-cyanopyridine provided an excellent PC yield of $>99 \%$ (chemo-selectivity $>99 \%$ ) in just $1 \mathrm{~h}$ using 20 mol\% catalyst $\left(\mathrm{CeO}_{2}\right)$, at $130{ }^{\circ} \mathrm{C} / 5 \mathrm{MPa} \mathrm{CO}$ and this is the highest yield of $\mathbf{P C}$ from $\mathrm{CO}_{2}$ and $\mathbf{P G}$ to date.

To extend the synthetic potential of this catalyst system, synthesis of six-membered ring carbonates was carried out by employing various 1,3-diols with monoalkyl-, dialkyl-, and phenyl-substitutions and generally the corresponding cyclic carbonates were obtained in high yields of 62-97\% with good to excellent chemo-selectivity (77-99\%). The ester that is formed by reaction of the diol starting material with 2-picolinamide in situ produced was spotted as the major byproduct. Syntheses of six-membered ring carbonates, especially those having multiple 
substituents, are difficult to realize using any methodology, despite the fact that they represent useful chemicals and intermediates for, inter alia, biodegradable polymers for drug delivery systems. The results obtained for this Ce-based catalyst system mediating the synthesis of various six-membered carbonates are highly attractive compared to other methodologies reported to date, except for the non-substituted trimethylene carbonate derived from oxetane and $\mathrm{CO}_{2}$, for which Kleij et al. [72] reported a very high yield (95\%) using a simple though highly reactive Al-catalyst based on amino-triphenolate ligands.

After the reaction, the $\mathrm{CeO}_{2}$ catalyst was removed from the reaction mixture and the filtrate was analyzed by coupled plasma atomic emission spectroscopy (ICP-AES), which indicated that no Ce species had leached into the filtrate $(<0.1 \mathrm{ppm})$. The catalyst was therefore successfully reused for three times without any loss of its high selectivity and yield; the BET surface area and X-ray diffraction pattern of the $\mathrm{CeO}_{2}$ material before and after the reaction remained virtually unchanged and thus indicated that the $\mathrm{CeO}_{2}$ catalyst is highly stable under the experimental conditions. The mechanistic details are similar to the ones already discussed for the formation of acyclic carbonates in section 1. Overall, this catalyst system shows the best yields for cyclic carbonate synthesis (five- and six-membered ones) from diols and $\mathrm{CO}_{2}$ reported to date.

\subsection{Organocatalysts}

Buchard and co-workers [73] performed the synthesis of six-membered cyclic carbonates directly from various 1,3-diols and $\mathrm{CO}_{2}$ at room temperature, $0.1 \mathrm{MPa}$ of $\mathrm{CO}_{2}$ using standard reagents. First, the selective mono-insertion of $\mathrm{CO}_{2}$ into one of the $\mathrm{O}-\mathrm{H}$ bonds of 1,3-butanediol in various solvents was examined in the presence of DBU as catalyst. After the selective formation of the mono-carbonate intermediate at low concentration, 1 equivalent of tosyl chloride/triethylamine was added to the reaction mixture and stirred at room temperature. The pure targeted product was isolated in an appreciable yield of $44 \%$, which was increased to $68 \%$ when a higher concentration of diol (going from 0.1 to $1.7 \mathrm{M}$ ) was applied. Investigation into the scope of diol substrates revealed that various 1,3-diols were good reaction partners in this organocatalytic approach, and the corresponding six-membered cyclic carbonates were isolated in low to good yields (11-70\%). After initial insertion of $\mathrm{CO}_{2}$, into one of the $\mathrm{O}-\mathrm{H}$ bonds, tosylation of the carbonate species or the remaining alcohol function can be envisaged. 
1 Hereafter, the cyclization proceeds via either an addition/elimination sequence or an $\mathrm{S}_{\mathrm{N}} 2$ pathway, leading to retention or inversion of stereochemistry, respectively.

However, the exclusive formation and isolation of the $(R, R)$-configured cyclic carbonate from $(R, R)-2-4-p e n t a n e d i o l$ (yield: 53\%) as well as the optical activities of the cyclic carbonates obtained from enantiopure $(R)$ - and $(S)$-1,3-butanediol, clearly indicated a preference for the addition/elimination pathway (Scheme 11), with no observable racemization or inversion of stereochemistry, which was further supported by DFT calculations.
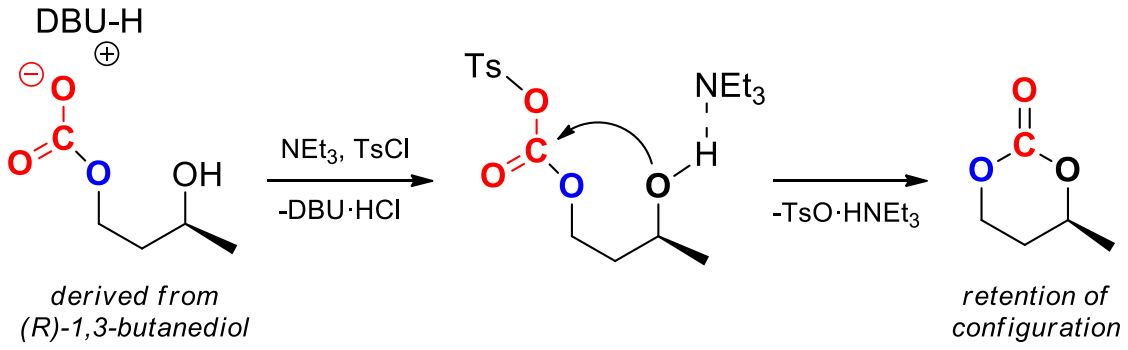

Scheme 11 Preferred pathway for the conversion of $(R)$-1,3-butanediol into its six-membered carbonate [73]

\section{Cyclic Carbonates derived from Unsaturated Alcohols}

\subsection{Metal based catalysts}

Reaction of $\mathrm{CO}_{2}$ with propargylic alcohols typically affords $\alpha$-alkylidene cyclic carbonates through a carboxylative cyclization process (Scheme 12), which is also a promising and green route to convert $\mathrm{CO}_{2}$. Moreover, $\alpha$-alkylidene cyclic carbonates possess a wide range of applications in organic synthesis being for instance building blocks in the formation of $\alpha$ hydroxy ketones and 5-methylene-oxazolidin-2-one derivatives.

Inoue et al. [74] performed the $\mathrm{Pd}(0)$-catalyzed $\left[\mathrm{Pd}\left(\mathrm{PPh}_{3}\right)_{4}\right]$ carboxylative cyclization reaction of $\mathrm{CO}_{2}(1 \mathrm{MPa})$ with sodium 2-methyl-3-butyn-2-olate (prepared from the corresponding alcohol and a slight excess of $\mathrm{NaH}$ ) and iodobenzene in THF as solvent at 100 ${ }^{\circ} \mathrm{C}$ and obtained the cyclic vinylidene carbonate in $68 \%$ yield. Alternatively, the use of copper catalysis proved to be highly beneficial to further develop this type of reaction. For instance, a cationic copper complex derived from 2,5,19,22-tetraaza[6,6](1,1')ferrocenophane-1,5-diene was an effective catalyst operating under $3.8 \mathrm{MPa}$ of $\mathrm{CO}_{2}$ and $100{ }^{\circ} \mathrm{C}$ affording good yields of 
the cyclic carbonates (>90\%) using various substituted propargylic alcohols under neat conditions [75]. Similarly, $\mathrm{CuCl}$ in the presence of the IL $[\mathrm{BMIm}]\left[\mathrm{PhSO}_{3}\right]$ yielded the $\alpha$ alkylidene cyclic carbonate in $97 \%$ yield under a milder $\mathrm{CO}_{2}$ pressure of $1 \mathrm{MPa}$ at $120{ }^{\circ} \mathrm{C}$ [76].

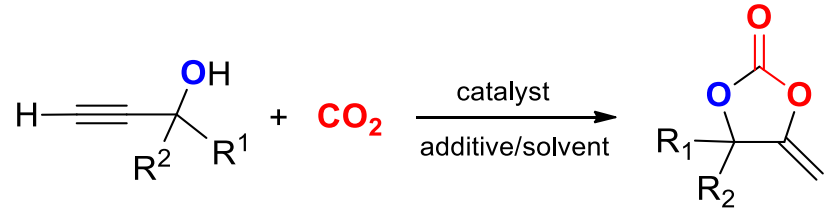

Scheme 12 Conversion of $\mathrm{CO}_{2}$ into an $\alpha$-alkylidene cyclic carbonate with an exo-cyclic double bond using propargylic alcohols

Substantial improvement in activity was reported for metal based catalyst systems when Mizuno et al. communicated a tungstate based complex (i.e., $\mathrm{TBA}_{2}\left[\mathrm{WO}_{4}\right], \mathrm{TBA}=$ tetrabutylammonium) as an efficient homogeneous catalyst for conversion of $\mathrm{CO}_{2}$ with propargylic alcohols to give the corresponding cyclic carbonates under mild reaction conditions [77]. DFT calculations allowed to optimize the tungstate structure and the basicities of oxygen atoms in various polyoxometalates (POMs) were compared with the natural bond orbital (NBO) charges; the simple $\left[\mathrm{WO}_{4}\right]^{2-}$ tungstate was found to be the most basic among the series. Under only $0.1 \mathrm{MPa}$ of $\mathrm{CO}_{2}$ pressure and at a relatively low temperature of $60{ }^{\circ} \mathrm{C}$, propargylic alcohol (2-methylbut-3-yn-2-ol) was coupled with $\mathrm{CO}_{2}$ in acetonitrile to give the corresponding carbonate structure in $76 \%$ yield. Upon lowering the catalyst loading while increasing the $\mathrm{CO}_{2}$ pressure ( $2 \mathrm{MPa}$ ) and reaction time, the total turnover number could be enhanced to a significant 473 with a yield of $95 \%$. In a similar way, more lethargic substrates such as propargylic alcohols with internal triple bonds ( $c f ., 1$-ethynylcyclohexan-1-ol) were also effectively transformed to their cyclic carbonates in excellent yield of up to $95 \%$.

Having witnessed the enhancement in activity brought about by the $\left[\mathrm{WO}_{4}\right]^{2-}$ anion in carbonate formation under much milder conditions, Song and co-workers [78] employed a $\mathrm{Ag}_{2} \mathrm{WO}_{4} / \mathrm{Ph}_{3} \mathrm{P}$ dual catalyst system for the conversion of $\mathrm{CO}_{2}$ and propargylic alcohols to provide the $\alpha$-alkylidene carbonates under solvent-free conditions. In this dual catalyst system, the $\left[\mathrm{WO}_{4}\right]^{2-}$ anion was envisioned to activate both $\mathrm{CO}_{2}$ and the propargylic alcohol generating a carboxylate intermediate which could then be intercepted by $\mathrm{Ag}$-activated $\mathrm{C} \equiv \mathrm{C}$ triple bond to afford the product (Scheme 13). 


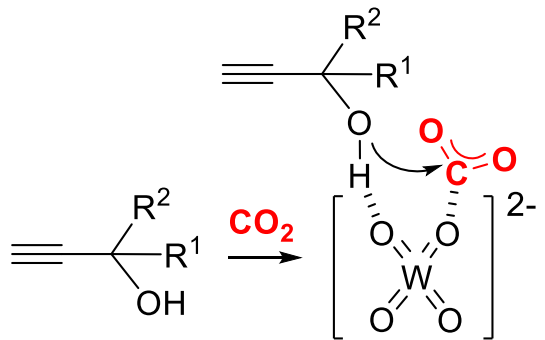

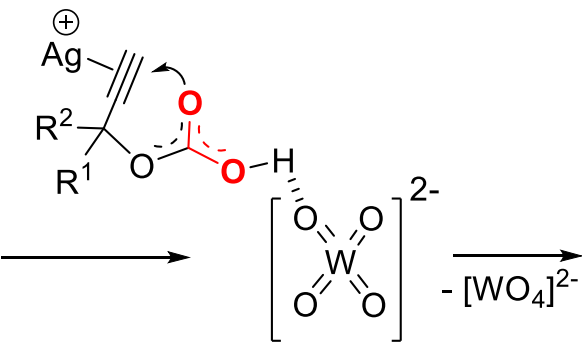

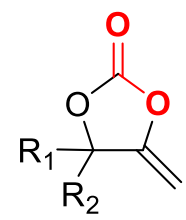

Scheme 13 Chemical fixation of $\mathrm{CO}_{2}$ through the dual activation pathway [78]

Using this system at $1 \mathrm{~mol} \%$ of catalyst loading, $25^{\circ} \mathrm{C}$ and $0.1 \mathrm{MPa}$ of $\mathrm{CO}_{2}$ pressure an optimum yield of $96 \%$ was obtained. Subsequently, other substrates were examined with this dual catalyst and propargylic alcohols with alkyl and aryl substituents at the propargylic position were also effective substrates to give the corresponding cyclic carbonates in good to excellent yields. The method was, however, not effective for 1-isopropyl and 1,1cyclopentylene substituted substrates which showed (very) low conversions due to a combination of steric hindrance and ring strain. Secondary propargylic alcohols failed to form the carbonate product, while the internal propargylic substrate 2-methyl-4-phenylbut-3-yn-2-ol required both higher $\mathrm{CO}_{2}$ pressure $(1 \mathrm{MPa})$ and temperature $\left(80^{\circ} \mathrm{C}\right)$ to afford the corresponding cyclic carbonate in good yield (82\%) using longer reaction times $(36 \mathrm{~h})$. This catalyst system was easily separated by extraction with hexane and after drying the catalyst could be successfully recycled four times without observable loss of activity.

At room temperature and $\mathrm{CO}_{2}$ pressures <2 $\mathrm{MPa}, \mathrm{AgOAc} / \mathrm{DBU}$ [79] and F-MOP-3-Ag/ DBU catalyst systems $(\mathrm{F}-\mathrm{MOPs}=$ fluorinated microporous organic polymers having $\mathrm{Ag}(\mathrm{I})$ sites incorporated) [80] in toluene gave good results. Notably, both catalyst systems were successful in converting various terminal and bulky internal propargylic alcohols to their corresponding cyclic carbonates in good yields. Heterogeneous metal systems have also been reported as effective catalysts for $\alpha$-alkylidene carbonate formation. Important limitations for these supported catalyst systems, though, were primarily the required supercritical conditions and high catalyst loadings and, moreover, a limited substrate scope allowing only the conversion of terminal propargylic alcohols [81, 82]. Significant improvement of activity and reusability features of heterogeneous metal catalysts was reported by Liu and co-workers [83] who used porous organic polymers (POPs) as a solid support. This material allows the introduction of various $\mathrm{CO}_{2}$-philic functional species inside its structure to obtain a more active, functional and reusable catalyst. Specifically, the authors prepared porous poly(triphenylphosphine) with azo 
$1 \quad(\mathrm{R}-\mathrm{N}=\mathrm{N}-\mathrm{R})$ functionalities (i.e., a poly $\left(\mathrm{PPh}_{3}\right)$-azo material) with the $\mathrm{Ag}$ sites being coordinated by the phosphine ligands. This system was efficiently used for $\mathrm{CO}_{2}$ transformations taking advantage of cooperative effects between the functional porous polymer and the metal species.

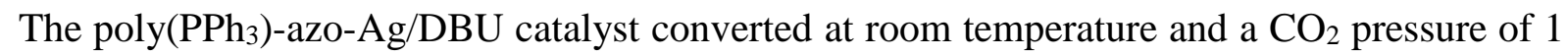
MPa the benchmark propargylic alcohol 2-methylbut-3-yn-2-ol to its corresponding cyclic carbonate with a yield of $56 \%$ in $3 \mathrm{~h}$; a higher yield was achieved by further increasing the reaction time to $18 \mathrm{~h}(>99 \%)$ with a remarkable total TON of 1563 . The presence of a high local concentration of $\mathrm{PPh}_{3}$ ligands and azo functionalities in the polymer $\left(\mathrm{PPh}_{3} / \mathrm{Ag}=200: 1, \mathrm{azo} / \mathrm{Ag}\right.$ $=300: 1)$ facilitated cooperative effects towards the formation of $\alpha$-alkylidene compounds. Moreover, the catalyst system was also shown to be recyclable at least five times without loss of activity. After several uses, transmission electron microscopic (TEM) analysis of the catalytic material indicated that the metallic Ag particles were still highly dispersed without changes in particle size after recycling. In order to establish possible leaching, the catalyst was separated through centrifugation after the reaction performed for $1 \mathrm{~h}$, and the filtrate was analyzed by ICP-OES which demonstrated that there was no observable leaching of Ag species ( $<10 \mathrm{ppb}$ ). Then, various terminal propargylic alcohols with both alkyl and aryl substituents were examined as substrates and these reacted efficiently with $\mathrm{CO}_{2}$, though substrates comprising bulky isopropyl groups or small rings required longer reaction times to obtain good yields presumably due to steric effects.

The proposed mechanism for the formation of $\alpha$-alkylidene cyclic carbonates mediated by the poly $\left(\mathrm{PPh}_{3}\right)$-azo-Ag catalyst system is shown in Scheme 14 [83]. First, the propargylic alcohol, activated by DBU, reacts with pre-activated $\mathrm{CO}_{2}$ to generate a carbonate intermediate. An intramolecular ring-closing step is then followed by proto-demetallation to afford the corresponding cyclic carbonate with the regeneration of the active Ag species and DBU.

Similarly, He et al. [84] used [ $\left.\left(\mathrm{PPh}_{3}\right)_{2} \mathrm{Ag}\right]_{2} \mathrm{CO}_{3}$ (in situ formed from $\mathrm{Ag}_{2} \mathrm{CO}_{3}$ and $\mathrm{PPh}_{3}$ ) as a robust and highly efficient single-component bifunctional catalyst for the coupling of propargylic alcohols and $\mathrm{CO}_{2}$ at room temperature and atmospheric pressure. After only $2 \mathrm{~h}, 2-$ methylbut-3-yn-2-ol and $\mathrm{CO}_{2}$ were converted to the cyclic carbonate in $93 \%$ yield under 


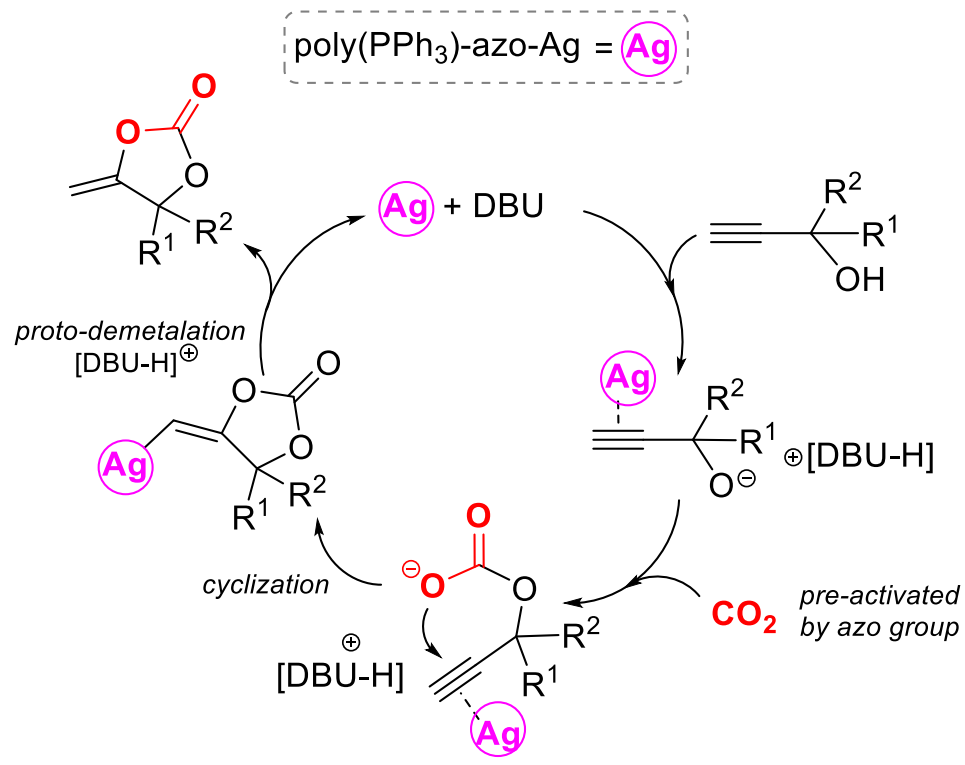

Scheme 14 Proposed mechanism for the coupling reaction of propargylic alcohols with $\mathrm{CO}_{2}$ catalyzed by poly(PPh3)-azo-Ag/DBU [83]

neat conditions using the in situ prepared catalyst. The recovered precipitate after the reaction could be reused in a subsequent carboxylative cyclization cycle.

Ag nanoparticles (NPs) have been immobilized to other solid supports such as sulfonated macro-reticular resins (SMRs) forming an active AgNPs/SMR catalyst [85]. Alternatively, Ag halides were supported on a porous carbon material $(\mathrm{AgX} @ \mathrm{C}, \mathrm{X}=\mathrm{Cl}, \mathrm{Br}$ and $\mathrm{I})$ [86] and the resultant catalyst was effective at room temperature and atmospheric pressure in the carboxylative cyclization of the propargylic alcohol 2-methylbut-3-yn-2-ol and $\mathrm{CO}_{2}$ in the presence of DBU as co-promoter. The use of the AgNPs/SMR catalyst gave a 91\% yield of the cyclic carbonate after $10 \mathrm{~h}$ in DMF, and the catalyst was shown to be recyclable at least five times. The AgI@C catalyst furnished the product in 99\% yield within $4 \mathrm{~h}$ in acetonitrile, and was used in a total of 10 cycles without any significant loss in activity.

Interestingly, a zinc salt $\left(\mathrm{ZnI}_{2}\right)$ in combination with triethylamine $\left(\mathrm{NEt}_{3}\right)$ was recently proposed as a catalyst system in this area and showed excellent synergistic effects to promote the solvent-free reaction of $\mathrm{CO}_{2}$ and 2-methylbut-3-yn-2-ol under a $\mathrm{CO}_{2}$ pressure of $1 \mathrm{MPa}$ and at $30{ }^{\circ} \mathrm{C}$ to obtain 4,4-dimethyl-5-methylene-1,3-dioxolan-2-one in 95\% yield after $10 \mathrm{~h}$ [87]. These results are promising to devise new catalyst systems based on earth-abundant and cheap metals such as Zn. 


\subsection{Organocatalysts}

As $\mathrm{CO}_{2}$ prevalently behaves as an electrophile, strong Lewis bases based on nitrogen heterocycles have the potential to activate $\mathrm{CO}_{2}$ affording zwitterionic adducts. Thus, such organocatalytic promotors can be utilized as convenient and cheap $\mathrm{CO}_{2}$ transformers to accomplish its conversion by increasing its nucleophilic character. $\mathrm{N}$-Heterocyclic carbenes (NHCs) incorporating electron-donating heteroatoms have a strong basic character and this enables strong $\sigma$-donor ability of the NHC useful for $\mathrm{CO}_{2}$ activation. Imidazolium-2carboxylates (with the integrated $\mathrm{CO}_{2}$ molecule in an activated state) have been spectroscopically and structurally identified as $\mathrm{NHC}-\mathrm{CO}_{2}$ adducts. Ikariya and co-workers [88] prepared NHCs (1,3-dialkylidazol-2-ylidenes) and their corresponding $\mathrm{CO}_{2}$ adducts (1,3dialkylimidazolium-2-carboxylates), and employed them as efficient catalysts for cyclic carbonate synthesis using propargylic alcohols and $\mathrm{CO}_{2}$.

The NHC- $-\mathrm{CO}_{2}$ adducts showed comparatively superior activity under milder conditions than the NHCs themselves, which required supercritical conditions to obtain good results for the conversion of 2-methyl-3propyn-2-ol in the carboxylative cyclization with $\mathrm{CO}_{2}$ to form the corresponding cyclic carbonate. Under solvent-free conditions at $4.5 \mathrm{MPa} \mathrm{CO}_{2}$ and $60{ }^{\circ} \mathrm{C}$, various $\mathrm{NHC}-\mathrm{CO}_{2}$ adducts, prepared by variation of the $\mathrm{N}$-substituents (di-isopropyl, di-tertbutyl, diaryl) were tested for their catalytic activity. The $\mathrm{NHC}-\mathrm{CO}_{2}$ adduct 1,3-di-tertbutylimidazolium-2-carboxylate gave the best yield (99\%) for the cyclic carbonate product among the adducts tested. If the catalyst loading, $\mathrm{CO}_{2}$ pressure and/or temperature were lowered, a significant amount of an acyclic product (1,1-dimethyl-2-oxo-propyl-1',1'dimethyl-2'-propynyl carbonate) was obtained along with the desired cyclic carbonate. The carboxylative cyclization affording the cyclic carbonate and the subsequent addition of another propargylic alcohol to the product is thought to lead to this $2: 1$ coupling product of both substrates.

Various five-membered cyclic carbonates were prepared in good yields from different propargylic substrates having disubstituted alkyne groups using the $\mathrm{NHC}-\mathrm{CO}_{2}$ catalyst. The presence of electron-withdrawing groups conjugated to the triple bond in the substrate led to the targeted products in faster rates and at lower reaction temperatures. The NHC-based catalyst also tolerates propargylic substrates equipped with heterocycles such as pyridine and thiophene, whereas allylic compounds such as 2-methyl-3-buten-2-ol and 2-methyl-4-phenyl-3-buten-2-ol did not give any cyclization product. In each product, the $\mathrm{C}=\mathrm{C}$ double bond was found to have a (Z)-configuration, indicating that the addition of the NHC-carboxylate to the alkyne fragment 
proceeded predominantly in an anti fashion. The postulated mechanism for the $\mathrm{NHC}-\mathrm{CO}_{2}$

2 mediated carboxylative cyclization of propargylic alcohols and $\mathrm{CO}_{2}$ involves the nucleophilic 3 addition of the imidazolium-2-carboxylate to the $\mathrm{C} \equiv \mathrm{C}$ triple bond and subsequent 4 intramolecular cyclization of the alkoxide intermediate (Scheme 15). A significant positive 5 effect of electron-donating $N$-alkyl substituents present in the NHC structure implies that the 6 intramolecular nucleophilic attack of the $\mathrm{CO}_{2}$ moiety, once bound to the NHC, onto the 7 substrates may be rate-limiting step in this catalytic cycle.

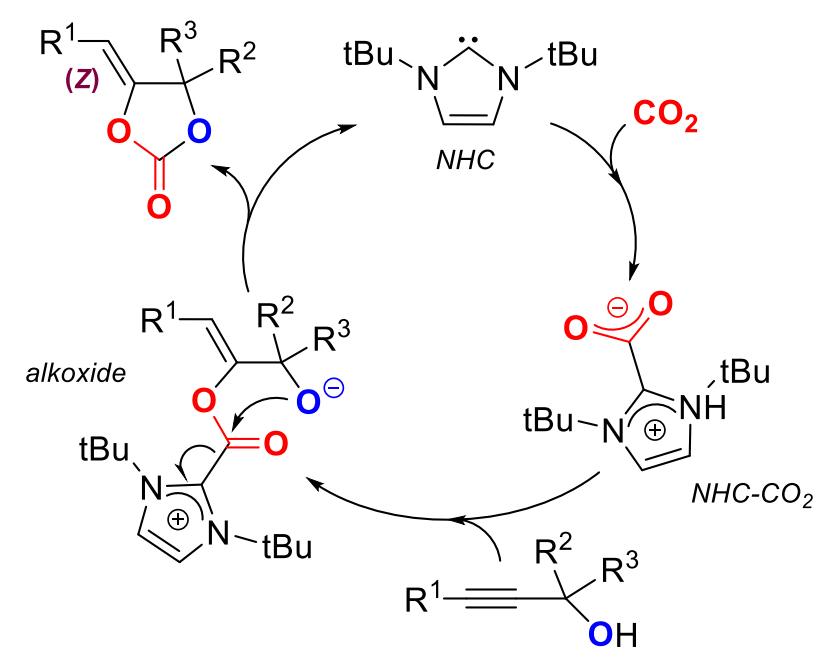

Scheme 15 Mechanism of the carboxylative cyclization catalyzed by a $\mathrm{NHC}-\mathrm{CO}_{2}$ adduct [88]

N-Heterocyclic olefins (NHOs) are compounds that are capable of further stabilizing the positive charge that arises upon activation of $\mathrm{CO}_{2}$ due to aromatization of the heterocyclic ring thereby making the terminal carbon atom of the initial olefin group more electronegative and susceptible towards the activation of electrophilic reaction partners. Recently, Lu et al. [89] prepared various $\mathrm{NHO}-\mathrm{CO}_{2}$ adducts and employed them as catalysts for coupling of propargylic alcohols and $\mathrm{CO}_{2}$ to yield $\alpha$-alkylidene carbonates. For comparative reasons, $\mathrm{NHC}-$ $\mathrm{CO}_{2}$ adducts were also prepared and in situ decarboxylation experiments monitored by IR spectroscopy in $\mathrm{CH}_{2} \mathrm{Cl}_{2}$ at $40{ }^{\circ} \mathrm{C}$ revealed that decarboxylation of the $\mathrm{NHO}$ systems occurred within $2 \mathrm{~h}$, whereas only small amounts of NHC adducts decomposed under similar conditions. This demonstrates that the decarboxylation of $\mathrm{NHO}-\mathrm{CO}_{2}$ adducts is relatively easy and therefore of more practical use for cyclic carbonate synthesis. The relatively poor thermal stability of $\mathrm{NHO}-\mathrm{CO}_{2}$ adducts therefore offers an opportunity to use these compounds as active catalysts for $\mathrm{CO}_{2}$ transformations at low temperature. In the catalyst activity screening phase, 
1 2-methyl-4-phenylbut-3-yn-2-ol was chosen as a model substrate and reacted with $\mathrm{CO}_{2}$ at 2

$2 \mathrm{MPa}$ pressure and $60{ }^{\circ} \mathrm{C}$ for $12 \mathrm{~h}$. Among the various $\mathrm{NHO}-\mathrm{CO}_{2}$ adducts, the isopropyl3 substituted NHO- $\mathrm{CO}_{2}$ adduct showed the best results leading to a $93 \%$ isolated yield of the 4 cyclic carbonate. The difference in catalytic activity observed for the $\mathrm{NHO}-\mathrm{CO}_{2}$ adduct in 5 comparison with its corresponding $\mathrm{NHC}-\mathrm{CO}_{2}$ was established for various propargylic substrates, and typically the NHO adducts are about 10-100 times more active than their NHC analogues. Various terminal and internal propargylic alcohols smoothly underwent the carboxylative cyclization reaction and were converted into their corresponding $\alpha$-alkylidene cyclic carbonates in moderate to excellent yields. Apparently only a slight structural difference exists between the NHO and $\mathrm{NHC}$ adducts; the much higher reactivity for the $\mathrm{NHO}-\mathrm{CO}_{2}$ adducts was tentatively ascribed to the lower stability of the $\mathrm{C}_{\text {carboxylate }}-\mathrm{C}_{\mathrm{NHO}}$ bond.

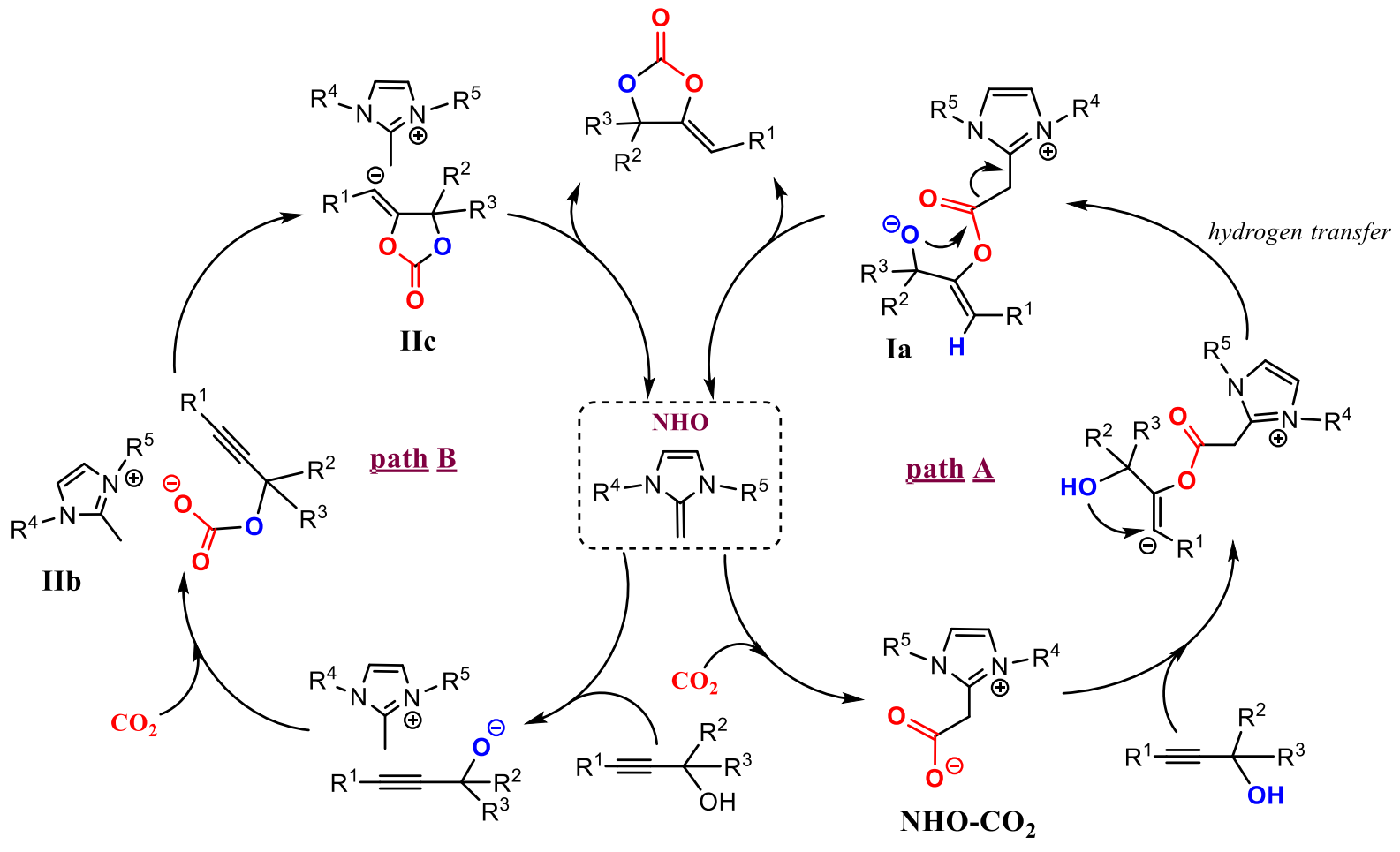

Scheme 16 Proposed mechanism of the carboxylation reaction catalyzed by the $\mathrm{NHO}-\mathrm{CO}_{2}$ adduct [89]

\section{The proposed mechanistic manifold begins with the zwitterionic compound $\mathrm{NHO}-\mathrm{CO}_{2}$ that} adds to the triple bond of propargylic substrate through nucleophilic attack. Meanwhile, hydrogen transfer of alcohol generates the new zwitterion Ia (Scheme 16, path A), and then the alkoxide anion attacks the carboxylate carbon to release the desired product and regenerating 
the $\mathrm{NHO}$, which rapidly captures free $\mathrm{CO}_{2}$ to form the $\mathrm{NHO}-\mathrm{CO}_{2}$ adduct to induce further turnover. The higher thermal instability of the NHO- $\mathrm{CO}_{2}$ adducts favorably adds to the overall kinetics of the reaction, thus creating higher turnover at lower temperatures as compared with the reactivity of analogous $\mathrm{NHC}-\mathrm{CO}_{2}$ adducts. The NHO with increased electronegativity at the terminal carbon atom can also act as a Brønsted base able to abstract a proton from the propargylic alcohol to form the intermediate IIa (path B) which subsequently reacts with $\mathrm{CO}_{2}$ to give intermediate IIb (Scheme 16, path B). Subsequently, the intermediate IIc is obtained by intramolecular ring-closure within intermediate IIb, which abstracts a proton from the 2methyl imidazolium cation to release the desired product. The obvious difference between both pathways $\mathrm{A}$ and $\mathrm{B}$ is that the hydrogen at the alkenyl position of cyclic carbonate originates exclusively from the propargylic substrate (path A) or both substrate and catalyst (path B): this aspect may be elucidated by a proper labeling of the NHO and/or propargylic substrate.

The same group also prepared various $\mathrm{CO}_{2}$ adducts of alkoxide-functionalized imidazolium betaines (abbreviated as AFIBs) and explored the AFIB- $\mathrm{CO}_{2}$ adducts as effective organocatalysts within the context of carboxylative cyclization of propargylic alcohols with $\mathrm{CO}_{2}$ [90]. The best result (97\% yield) for the AFIB- $-\mathrm{CO}_{2}$ mediated formation of the cyclic carbonate product was obtained under $2 \mathrm{MPa}$ pressure at $60{ }^{\circ} \mathrm{C}$ using 2-methylbut-3-yn-2-ol as substrate. The catalyst system proved to be more effective for the carboxylative cyclization of terminal rather than internal propargylic substrates.

Minakata et al. [91] treated various allylic alcohols with stoichiometric $t \mathrm{BuOI}$ under $0.1 \mathrm{MPa}$ of $\mathrm{CO}_{2}$ pressure and low reaction temperature resulting in the synthesis of five-membered cyclic carbonates containing a potentially useful alkyliodide group. The reagent, $t \mathrm{BuOI}$, can be readily prepared in situ from commercially available tert-butyl hypochlorite $(t \mathrm{BuOCl})$ and sodium iodide (NaI), and serves to iodinate an elusive and rather unstable alkyl carbonic acid that is first generated from $\mathrm{CO}_{2}$ and an unsaturated alcohol. The introduction of the iodine atom radically changes the position of the equilibrium of the initial $\mathrm{CO}_{2}$-trapping reaction (Scheme 17). The use of tetrahydrofuran (THF) as solvent and a reaction temperature of $-20{ }^{\circ} \mathrm{C}$ resulted in the conversion of prop-2-en-1-ol into the corresponding cyclic carbonate in $92 \%$ yield. To further investigate the efficacy of $t \mathrm{BuOI}$, other iodinating reagents such as bis(pyridine)iodine tetrafluroborate $\left(\mathrm{IPy}_{2} \mathrm{BF}_{4}\right), N$-iodosuccinimide (NIS), $\mathrm{I}_{2}$ and a combination of $\mathrm{I}_{2}$ and triethylamine were tested, but all these reagents failed to provide the desired product.

The main reason for $t \mathrm{BuOI}$ being the most appropriate iodinating reagent is related to the liberation of a relatively weak acid (i.e., $t \mathrm{BuOH}$ ) during the reaction of allyl-carbonic acid and 
$1 \quad t$ BuOI. $\beta$-Branched allylic alcohols also could be smoothly transformed into their corresponding cyclic carbonates in good yields. Both $(E)$ - and $(Z)$-allylic alcohols were transformed into their corresponding cyclic carbonates. Allyl alcohols containing rigid cyclic olefins, hydroxyl, ester or silyl groups, and homo-allylic alcohols allowing the formation of six-membered cyclic carbonates were also compatible with this $\mathrm{CO}_{2}$ conversion reaction. Similarly, various internal propargylic alcohols reacted with $\mathrm{CO}_{2}$ under similar conditions and permitted the synthesis of the corresponding iodoalkyl derived carbonates in good yields.

(a) $\mathrm{H}-\mathrm{A}+\mathrm{t}-\mathrm{BuOI} \longrightarrow \mathrm{I}-\mathrm{A}+\mathrm{t}-\mathrm{BuOH}$

(b)

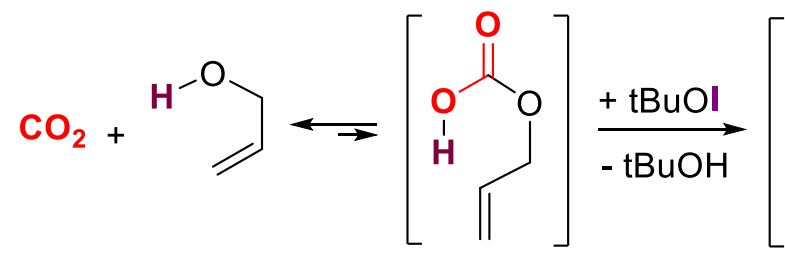

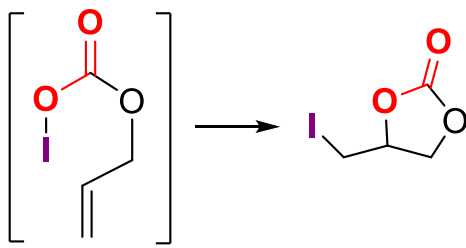

Scheme 17 (a) Reaction of tert-butyl hypoiodite with weak acids. (b) Strategy for trapping carbonic acids with tert-butyl hypoiodite [91]

Recently, an efficient carboxylation/alkene functionalization reaction of homoallylic alcohols was reported by Johnston et al. to produce chiral cyclic carbonates (Scheme 18) [92] using an approach similar to the one reported by Minakata (vide supra). At low temperatures and ambient pressure, a toluene solution of 3-phenylbut-3-en-1-ol was treated with $\mathrm{CO}_{2} / \mathrm{N}$ iodosuccinimide in the presence of various bases including NaH, DBU, TBD, DMAP as well as hydrogen bond donors such as TFA and thiourea but these conditions failed to deliver the desired carbonate product or gave only rise to low yields. A Brønsted acid/base combination was then explored to promote the reaction and the use of a chiral pyrrolidine-substituted bis(amidine) gave a promising $18 \%$ yield of the iodinated cyclic carbonate in $39 \%$ ee. An analogous catalyst incorporating trans-stilbene diamine (StilbPBAM; Scheme 18) instead of trans-cyclohexane diamine provided the product in $33 \%$ yield and $36 \%$ ee. Exploration of strong Brønsted acid additives $\left(\mathrm{HNTf}_{2}\right.$ ) (1 equiv) combined with the StilbPBAM organocatalyst enhanced the activity to provide a $95 \%$ yield $(91 \% e e)$ in the presence of molecular sieves (4 $\AA$ ). Combined, the results suggest an important role for hydrogen-bonding in the key selectivity-determining step, and a unique reactivity associated with the proper mutual positioning of the Brønsted acid and base in the relevant transition state that controls the 
asymmetric induction. Various other substituted styrene homoallylic alcohols were also tested: $\beta$-naphthyl substituted anisole derivatives (meta- and para-substituted), and halogen substituted substrates were all converted into their six-membered carbonates with excellent enantioselectivity and in good yields, whereas substitution near the alkene moiety was not tolerated and no conversion was noted in these cases.
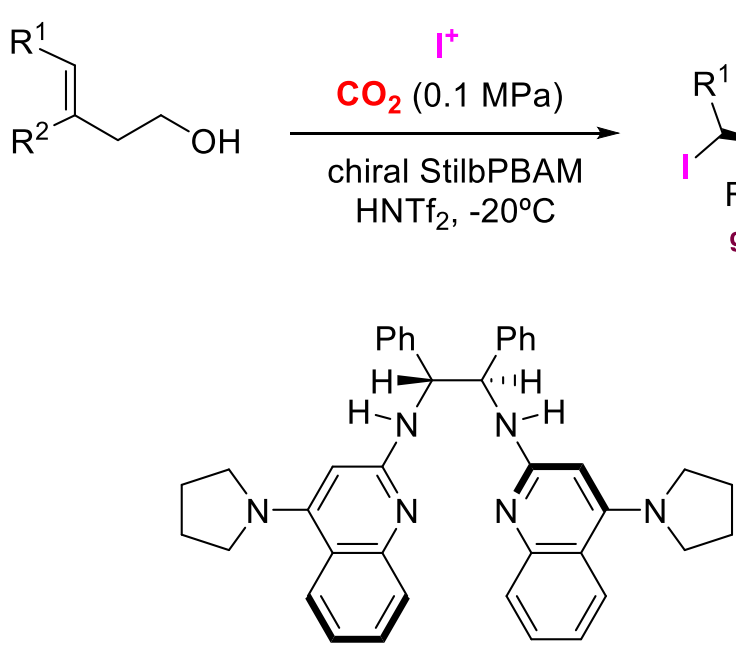

StilbPBAM

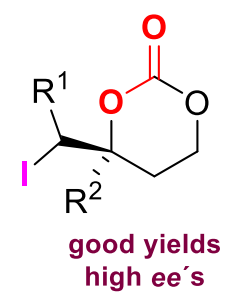

high ee's

Scheme 18 An enantioselective method for the synthesis of cyclic carbonates from homoallylic alcohols and $\mathrm{CO}_{2}$ using a chiral StilbPBAM organocatalyst [92]

\section{Cyclic Carbonates from Halo-Alcohols}

Another approach for the preparation of cyclic carbonates from $\mathrm{CO}_{2}$ could be the use of halohydrins (haloalcohols) as potential starting substrate in presence of a base. The obvious drawback of this approach is the stoichiometric amount of halide waste that is produced alongside, but in certain cases it may provide an alternative if other methods fail to deliver the desired organic carbonate structure.

Using PEG-400 as an environmentally friendly solvent and $\mathrm{K}_{2} \mathrm{CO}_{3}$ as an easily accessible base, various cyclic carbonates (yield 72-100\%) were prepared by the group of $\mathrm{Wu}$ starting from vicinal halohydrins such as chlorohydrin, bromohydrin, and phenyl and alkyl-substituted halohydrins at low $\mathrm{CO}_{2}$ pressure $(2 \mathrm{MPa})$ and temperature $\left(50{ }^{\circ} \mathrm{C}\right)$ [93]. The choice of PEG400 as a reaction medium is beneficial in terms of solvation of the potassium cation to increase the basicity of $\mathrm{K}_{2} \mathrm{CO}_{3}$, an increase in $\mathrm{CO}_{2}$ concentration in this specific medium accelerating the reaction, and the ease of product separation. Similarly, Zhang and co-workers [94] reported 
on the utilization of 1.1 equiv. of $\mathrm{Cs}_{2} \mathrm{CO}_{3}$ as base, obtaining both five- and six-membered cyclic carbonates in good to excellent yield under relatively mild conditions $\left(40{ }^{\circ} \mathrm{C}\right.$ under $0.1 \mathrm{MPa}$ $\mathrm{CO}_{2}$, Scheme 19). By variation of different solvents and bases, the optimal conditions were determined $\left(\mathrm{Cs}_{2} \mathrm{CO}_{3}, \mathrm{DMF}\right)$ and the haloalcohol substrate 3-chloro-1-propanol reacted efficiently with $\mathrm{CO}_{2}$ giving $95 \%$ yield of trimethylene carbonate (TMC). One advantage of this method, which seems a general approach if the haloalcohol is readily available, is the easy formation of larger-ring size cyclic carbonates which remains a synthetic challenge in the area of organic carbonates. The mechanism of this reaction is pretty straightforward with the $\mathrm{Cs}_{2} \mathrm{CO}_{3}$ first deprotonating the alcohol resulting in the formation of a cesium alkoxide. The latter in turn reacts with $\mathrm{CO}_{2}$ to form a carbonate intermediate which in the final step undergoes an intramolecular ring closing reaction affording $\mathrm{CsCl}$ as a byproduct and TMC.
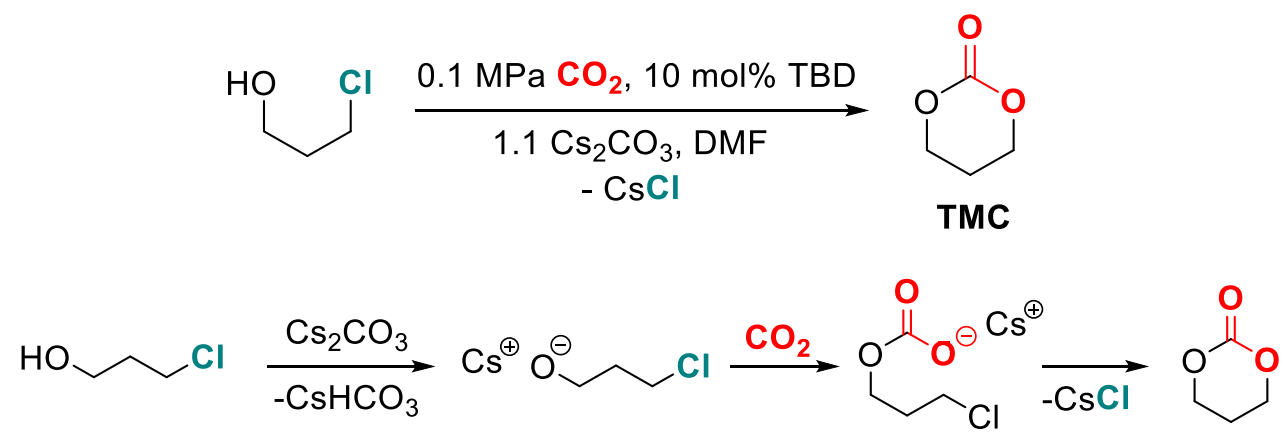

Scheme 19 Reaction manifold for the formation of TMC using 3-chloro-1-propanol as the starting material and $\mathrm{Cs}_{2} \mathrm{CO}_{3}$ as base [94]

Even more recently, Buchard and coworkers developed a related protocol for the formation of six-membered cyclic carbonates starting from 1,3-diols at low (0.1 $\mathrm{MPa}) \mathrm{CO}_{2}$ pressure and using DBU [95]. The procedure involves the activation of both the alcohol functions of the substrate by tosyl chloride and DBU allowing the in situ formation of a pseudo haloalcohol. This intermediate is then easily converted in the presence of $\mathrm{NEt}_{3}$ to the desired cyclic carbonate. DFT analysis revealed that the mechanism most likely goes through an addition/elimination sequence with intermediate formation of a tosylated carbonate species, and subsequent attack of the other activated alcohol (by $\mathrm{NEt}_{3}$ ) onto this carbonate fragment releasing the product with retention of configuration as was indeed experimentally observed for various chiral substrates. As for the aforementioned methodologies, stoichiometric amounts of 
1 DBU-HCl and $\mathrm{TsOHNEt}_{3}$ are produced, and some optimization regarding the atom-economy

2 will still be required.

\section{Conclusions and Outlook}

5 This overview of the latest developments in the area of cyclic carbonate synthesis from alcohol substrates and $\mathrm{CO}_{2}$ shows several advances made over the last 5-10 years with a major focus on the more recent achievements. Since the direct conversion of alcohols in the presence of $\mathrm{CO}_{2}$ is thermodynamically limited and only low equilibrium yields can be attained, several catalytic processes have been developed to circumvent this issue by using dehydrating agents. This approach has resulted in the high yield synthesis of both cyclic as well as acyclic carbonates in good yields, though a crucial feature to optimize remains the regeneration of the dehydrating species. If this agent can be efficiently recycled then such a process would be extremely useful for larger scale preparation of carbonates, and commercial exploitation. Apart from bypassing the thermodynamic limitations, other approaches that use more functional substrates such as propargylic alcohols have also been proven to be effective, and the carboxylative cyclization reaction is now a valuable tool in organic synthesis. Similar types of activation protocols where the initial alcohol function can first react with $\mathrm{CO}_{2}$ to form a linear carbonate followed by intramolecular attack onto a pre-activated alkyne fragment would be welcome in order to design new conversions and amplify the role of mono- and polyalcohols as suitable platform molecules in organic synthesis. Several useful protocols towards five- and six-membered carbonate synthesis in the presence of stoichiometric amounts of often simple and cheap reagents have already been developed. However, to answer to ever-growing need for more sustainable manufacturing of bulk and fine chemicals, new catalysis protocols are warranted to address this feature more effectively. Thus it seems that the combination of (poly)alcohols and $\mathrm{CO}_{2}$ as reaction partners will continue to inspire scientists to work on greener and more sustainable catalytic methods for the production of carbonated compounds that will find use in areas diverse as bulk, polymer and pharmaceutical chemistry. 


\section{References}

1. Huang S, Yan B, Wang S, Ma X (2015) Recent advances in dialkyl carbonates synthesis and applications. Chem Soc Rev 44:3079-3116.

2. Aresta M, Dibenedetto A, Dutta A (2016) Energy issues in the utilization of $\mathrm{CO}_{2}$ in the synthesis of chemicals: The case of the direct carboxylation of alcohols to dialkylcarbonates. Catal Today in press. doi: 10.1016/j.cattod.2016.02.046

3. Tundo P, Selva M (2002) The chemistry of dimethyl carbonate. Acc Chem Res 35:706716.

4. Pacheco MA, Marshall CL (1997) Review of Dimethyl Carbonate (DMC) Manufacture and Its Characteristics as a Fuel Additive. Energy \& Fuels 11:2-29.

5. Shaikh A-AG (1996) Organic Carbonates. Chem Rev 96:951-976.

6. Choi J-C, He L-N, Yasuda H, Sakakura T (2002) Selective and high yield synthesis of dimethyl carbonate directly from carbon dioxide and methanol. Green Chem 4:230-234.

7. Honda M, Tamura M, Nakagawa Y, Tomishige K (2014) Catalytic $\mathrm{CO}_{2}$ conversion to organic carbonates with alcohols in combination with dehydration system. Catal Sci Technol 4:2830-2845.

8. Shukla K, Srivastava VC (2016) Diethyl carbonate: critical review of synthesis routes, catalysts used and engineering aspects. RSC Adv 6:32624-32645.

9. Gong J, Ma X, Wang S (2007) Phosgene-free approaches to catalytic synthesis of diphenyl carbonate and its intermediates. Appl Catal A Gen 316:1-21.

10. Aresta M, Dibenedetto A, Fracchiolla E, Giannoccaro P, Pastore C, Pápai I, Schubert G (2005) Mechanism of formation of organic carbonates from aliphatic alcohols and carbon dioxide under mild conditions promoted by carbodiimides. DFT calculation and experimental study. J Org Chem 70:6177-6186.

11. Chaturvedi D, Mishra N, Mishra V (2007) A high yielding, one-pot synthesis of dialkyl carbonates from alcohols using Mitsunobu's reagent. Tetrahedron Lett 48:5043-5045.

12. Lim YN, Lee C, Jang H-Y (2014) Metal-Free Synthesis of Cyclic and Acyclic Carbonates from $\mathrm{CO}_{2}$ and Alcohols. Eur J Org Chem 1823-1826. 
1 13. Bloodworth BAJ, Davies AG, Vasishtha SC (1967) Organometallic Reactions. Part VII. Further Addition Reactions of Tributyltin Methoxide and of Bistributyltin Oxide. J Chem Soc C 1309-1313.

14. Hidai M, Hikita T, Uchida Y (1972) Reactions of Carbon Dioxide with Transition Metal Alkoxides. Chem Lett 521-522.

15. Choi J-C, Kohno K, Ohshima Y, Yasuda H, Sakakura T (2008) Tin- or titaniumcatalyzed dimethyl carbonate synthesis from carbon dioxide and methanol: Large promotion by a small amount of triflate salts. Catal Commun 9:1630-1633.

16. Kohno K, Choi J-C, Ohshima Y, Yasuda H, Sakakura T (2008) Synthesis of dimethyl carbonate from carbon dioxide catalyzed by titanium alkoxides with polyether-type ligands. ChemSusChem 1:186-188.

17. Aresta M, Dibenedetto A, Pastore C (2003) Synthesis and Characterization of $\mathrm{Nb}(\mathrm{OR})_{4}[\mathrm{OC}(\mathrm{O}) \mathrm{OR}](\mathrm{R}=\mathrm{Me}, \mathrm{Et}, \mathrm{Allyl})$ and Their Reaction with the Parent Alcohol To Afford Organic Carbonates. Inorg Chem 42:3256-3261.

18. Kizlink J, Pastucha I (1995) Preparation of Dimethyl Carbonate from Methanol and Carbon Dioxidee in the presence of Sn(IV) and Ti(IV) Alkoxides and Metal Acetates. Collect Czechoslov Chem Commun 60:687-692.

19. Kizlink J (1993) Synthesis of Dimethyl Carbonate from Carbon Dioxide and Methanol in the Presence of Organotin Compounds. Collect Czechoslov Chem Commun 58:13991402.

20. Kizlink J, Pastucha I (1994) Preparation of Dimethyl Carbonate from Methanol and Carbon Dioxide in the presence of Organotin Compounds. Collect Czechoslov Chem Commun 59:2116-2118.

21. Sakakura T, Saito Y, Okano M, Choi J-C, Sako T (1998) Selective Conversion of Carbon Dioxide to Dimethyl Carbonate by Molecular. J Org Chem 63:7095-7096.

22. Sakakura T, Choi J, Saito Y, Masuda T, Sako T, Oriyama T (1999) Metal-Catalyzed Dimethyl Carbonate Synthesis from Carbon Dioxide and Acetals. J Org Chem 64:45064508.

23. Choi J-C, Sakakura T, Sako T (1999) Reaction of Dialkyltin Methoxide with Carbon 
Dioxide Relevant to the Mechanism of Catalytic Carbonate Synthesis. J Am Chem Soc 121:3793-3794.

24. Sakakura T, Choi J-C, Saito Y, Sako T (2000) Synthesis of dimethyl carbonate from carbon dioxide: catalysis and mechanism. Polyhedron 19:573-576.

25. Kohno K, Choi J-C, Ohshima Y, Yili A, Yasuda H, Sakakura T (2008) Reaction of dibutyltin oxide with methanol under $\mathrm{CO}_{2}$ pressure relevant to catalytic dimethyl carbonate synthesis. J Organomet Chem 693:1389-1392.

26. Wakamatsu K, Orita A, Otera J (2010) DFT Study on Activation of Carbon Dioxide by Dimethytin Dimethoxide for Synthesis of Dimethyl Carbonate. Organometallics 29:1290-1295.

27. Stoian DC, Taboada E, Llorca J, Molins E, Medina F, Segarra AM (2013) Boosted $\mathrm{CO}_{2}$ reaction with methanol to yield dimethyl carbonate over $\mathrm{Mg}$-Al hydrotalcite-silica lyogels. Chem Commun 49:5489-5491.

28. Kumar S, Khatri OP, Cordier S, Boukherroub R, Jain SL (2015) Graphene oxide supported molybdenum cluster: first heterogenized homogeneous catalyst for the synthesis of dimethylcarbonate from $\mathrm{CO}_{2}$ and methanol. Chem Eur J 21:3488-3494.

29. Wu XL, Xiao M, Meng YZ, Lu YX (2005) Direct synthesis of dimethyl carbonate on $\mathrm{H}_{3} \mathrm{PO}_{4}$ modified $\mathrm{V}_{2} \mathrm{O}_{5}$. J Mol Catal A Chem 238:158-162.

30. Wu XL, Meng YZ, Xiao M, Lu YX (2006) Direct synthesis of dimethyl carbonate (DMC) using Cu-Ni/VSO as catalyst. J Mol Catal A Chem 249:93-97.

31. Tomishige K, Sakaihori T, Ikeda Y, Fujimoto K (1999) A novel method of direct synthesis of dimethyl carbonate from methanol and carbon dioxide catalyzed by zirconia. Catal Letters 58:225-229.

32. Ikeda Y, Sakaihori T, Tomishige K, Fujimoto K (2000) Promoting effect of phosphoric acid on zirconia catalysts in selective synthesis of dimethyl carbonate from methanol and carbon dioxide. Catal Letters 66:59-62.

33. Tomishige K, Ikeda Y, Sakaihori T, Fujimoto K (2000) Catalytic properties and structure of zirconia catalysts for direct synthesis of dimethyl carbonate from methanol and carbon dioxide. J Catal 192:355-362. 
34. Tomishige K, Furusawa Y, Ikeda Y, Asadullah M, Fujimoto K (2001) $\mathrm{CeO}_{2}-\mathrm{ZrO}_{2}$ solid solution catalyst for selective synthesis of dimethyl carbonate from methanol and carbon dioxide. Catal Letters 76:71-74.

35. Yoshida Y, Arai Y, Kado S, Kunimori K, Tomishige K (2006) Direct synthesis of organic carbonates from the reaction of $\mathrm{CO}_{2}$ with methanol and ethanol over $\mathrm{CeO}_{2}$ catalysts. Catal Today 115:95-101.

36. Ikeda Y, Asadullah M, Fujimoto K, Tomishige K (2001) Structure of the Active Sites on $\mathrm{H}_{3} \mathrm{PO}_{4} / \mathrm{ZrO}_{2}$ Catalysts for Dimethyl Carbonate Synthesis from Methanol and Carbon Dioxide. J Phys Chem B 105:10653-10658.

37. Lee HJ, Joe W, Song IK (2011) Direct synthesis of dimethyl carbonate from methanol and carbon dioxide over transition metal oxide/Ce ${ }_{0.6} \mathrm{Zr}_{0.4} \mathrm{O}_{2}$ catalysts: Effect of acidity and basicity of the catalysts. Korean J Chem Eng 29:317-322.

38. Jiang C, Guo Y, Wang C, Hu C, Wu Y, Wang E (2003) Synthesis of dimethyl carbonate from methanol and carbon dioxide in the presence of polyoxometalates under mild conditions. Appl Catal A Gen 256:203-212.

39. Honda M, Suzuki A, Noorjahan B, Fujimoto K, Suzuki K, Tomishige K (2009) Low pressure $\mathrm{CO}_{2}$ to dimethyl carbonate by the reaction with methanol promoted by acetonitrile hydration. Chem Commun 4596-4598.

40. Honda M, Kuno S, Begum N, Fujimoto K, Suzuki K, Nakagawa Y, Tomishige K (2010) Catalytic synthesis of dialkyl carbonate from low pressure $\mathrm{CO}_{2}$ and alcohols combined with acetonitrile hydration catalyzed by $\mathrm{CeO}_{2}$. Appl Catal A 384: 165-170.

41. Honda M, Kuno S, Sonehara S, Fujimoto K, Suzuki K, Nakagawa Y, Tomishige K (2011) Tandem Carboxylation-Hydration Reaction System from Methanol, $\mathrm{CO}_{2}$ and Benzonitrile to Dimethyl Carbonate and Benzamide Catalyzed by $\mathrm{CeO}_{2}$. ChemCatChem $3: 365-370$.

42. Tamura M, Wakasugi H, Shimizu K, Satsuma A (2011) Efficient and substrate-specific hydration of nitriles to amides in water by using a $\mathrm{CeO}_{2}$ catalyst. Chem Eur J 17:1142811431.

43. Honda M, Tamura M, Nakagawa Y, Sonehara S, Suzuki K, Fujimoto K, Tomishige K (2013) Ceria-catalyzed conversion of carbon dioxide into dimethyl carbonate with 2- 
cyanopyridine. ChemSusChem 6:1341-1344.

44. Honda M, Tamura M, Nakagawa Y, Nakao K, Suzuki K, Tomishige K (2014) Organic carbonate synthesis from $\mathrm{CO}_{2}$ and alcohol over $\mathrm{CeO}_{2}$ with 2-cyanopyridine: Scope and mechanistic studies. J Catal 318:95-107.

45. Jung KT, Bell AT (2002) Effects of catalyst phase structure on the elementary processes involved in the synthesis of dimethyl carbonate from methanol and carbon dioxide over zirconia. Top Catal 20:97-105.

46. Tamura M, Kishi R, Nakagawa Y, Tomishige K (2015) Self-assembled hybrid metal oxide base catalysts prepared by simply mixing with organic modifier. Nature Commun 6:8580.

47. Tamura M, Sawabe K, Tomishige K, Satsuma A, Shimizu K (2015) Substrate-Specific Heterogeneous Catalysis of $\mathrm{CeO}_{2}$ by Entropic Effects via Multiple Interactions. ACS Catal 5: 20-26.

48. Aresta M, Dibenedetto A, Pastore C, Angelini A, Aresta B, Pápai I (2010) Influence of $\mathrm{Al}_{2} \mathrm{O}_{3}$ on the performance of $\mathrm{CeO}_{2}$ used as catalyst in the direct carboxylation of methanol to dimethylcarbonate and the elucidation of the reaction mechanism. J Catal 269:44-52.

49. Chen L, Wang S, Zhou J, Shen Y, Zhao Y, Ma X (2014) Dimethyl carbonate synthesis from carbon dioxide and methanol over $\mathrm{CeO}_{2}$ versus over $\mathrm{ZrO}_{2}$ : comparison of mechanisms. RSC Adv 4:30968-30975.

50. Bansode A, Urakawa A (2014) Continuous DMC synthesis from $\mathrm{CO}_{2}$ and methanol over a $\mathrm{CeO}_{2}$ catalyst in a fixed bed reactor in the presence of a dehydrating agent. ACS Catal 4:3877-3880.

51. Stoian D, Bansode A, Medina F, Urakawa A (2016) Catalysis under microscope: Unraveling the mechanism of catalyst de- and re-activation in the continuous dimethyl carbonate synthesis from $\mathrm{CO}_{2}$ and methanol in the presence of a dehydrating agent. Catal Today in press. doi: 10.1016/j.cattod.2016.03.038

52. Honda M, Tamura M, Nakao K, Suzuki K, Nakagawa Y, Tomishige K (2014) Direct Cyclic Carbonate Synthesis from $\mathrm{CO}_{2}$ and Diol over Carboxylation/Hydration Cascade Catalyst of $\mathrm{CeO}_{2}$ with 2-Cyanopyridine. ACS Catal 4:1893-1896. 
53. Honda M, Sonehara S, Yasuda H, Nakagawa Y, Tomishige K (2011) Heterogeneous $\mathrm{CeO} 2$ catalyst for the one-pot synthesis of organic carbamates from amines, $\mathrm{CO}_{2}$ and alcohols. Green Chem 13: 3406-3413.

54. Tamura M, Honda M, Noro K, Nakagawa Y, Tomishige K (2013) Heterogeneous $\mathrm{CeO}_{2-}$ catalyzed selective synthesis of cyclic carbamates from $\mathrm{CO}_{2}$ and aminoalcohols in acetonitrile solvent. J Catal 305: 191-203.

55. Tamura M, Noro K, Honda M, Nakagawa Y, Tomishige K (2013) Highly efficient synthesis of cyclic ureas from $\mathrm{CO}_{2}$ and diamines by a pure $\mathrm{CeO}_{2}$ catalyst using a 2propanol solvent. Green Chem 15: 1567-1577.

56. Tamura M, Ito K, Honda M, Nakagawa Y, Sugimoto H, Tomishige K (2016) Direct Copolymerization of $\mathrm{CO}_{2}$ and Diols. Sci Rep 6:24038.

57. Tamura M, Honda M, Nakagawa Y, Tomishige K (2014) Direct conversion of $\mathrm{CO}_{2}$ with diols, aminoalcohols and diamines to cyclic carbonates, cyclic carbamates and cyclic ureas using heterogeneous catalysts. J Chem Technol Biotechnol 89: 19-33.

58. Tomishige K, Yasuda H, Yoshida Y, Nurunnabi M, Li B, Kunimori K (2004) Catalytic performance and properties of ceria based catalysts for cyclic carbonate synthesis from glycol and carbon dioxide. Green Chem 6:206-214.

59. Tomishige K, Yasuda H, Yoshida Y, Nurunnabi M, Li B, Kunimori K (2004) Novel route to propylene carbonate: selective synthesis from propylene glycol and carbon dioxide. Catal Letters 95: 45-49.

60. Du Y, He L-N, Kong D-L (2008) Magnesium-catalyzed synthesis of organic carbonate from 1,2-diol/alcohol and carbon dioxide. Catal Commun 9:1754-1758.

61. Du Y, Kong D-L, Wang H-Y, Cai F, Tian J-S, Wang J-Q, He L-N (2005) Sn-catalyzed synthesis of propylene carbonate from propylene glycol and $\mathrm{CO}_{2}$ under supercritical conditions. J Mol Catal A Chem 241:233-237.

62. Huang S, Liu S, Li J, Zhao N, Wei W, Sun Y (2006) Effective synthesis of propylene carbonate from propylene glycol and carbon dioxide by alkali carbonates. Catal Letters 112:187-191.

63. Vieville C, Yoo JW, Pelet S, Mouloungui Z (1998) Synthesis of glycerol carbonate by 
direct carbonatation of glycerol in supercritical $\mathrm{CO}_{2}$ in the presence of zeolites and ion exchange resins. Catal Letters 56:245-247.

64. Aresta M, Dibenedetto A, Nocito F, Pastore C (2006) A study on the carboxylation of glycerol to glycerol carbonate with carbon dioxide: The role of the catalyst, solvent and reaction conditions. J Mol Catal A Chem 257:149-153.

65. Dibenedetto A, Angelini A, Aresta M, Ethiraj J, Fragale C, Nocito F (2011) Converting wastes into added value products: From glycerol to glycerol carbonate, glycidol and epichlorohydrin using environmentally friendly synthetic routes. Tetrahedron 67:13081313.

66. George J, Patel Y, Pillai SM, Munshi P (2009) Methanol assisted selective formation of 1,2-glycerol carbonate from glycerol and carbon dioxide using $\mathrm{nBu}_{2} \mathrm{SnO}$ as a catalyst. $\mathrm{J}$ Mol Catal A Chem 304:1-7.

67. Li H, Gao D, Gao P, Wang F, Zhao N, Xiao F, Wei W, Sun Y (2013) The synthesis of glycerol carbonate from glycerol and $\mathrm{CO}_{2}$ over $\mathrm{La}_{2} \mathrm{O}_{2} \mathrm{CO}_{3}-\mathrm{ZnO}$ catalysts. Catal Sci Technol 3:2801-2809.

68. Zhang J, He D (2014) Surface Properties of $\mathrm{Cu} / \mathrm{La}_{2} \mathrm{O}_{3}$ and Its Catalytic Performance in the Synthesis of Glycerol Carbonate and Monoacetin from Glycerol and Carbon Dioxide. J Colloid Interface Sci 419:31-38.

69. Zhang J, He D (2015) Synthesis of glycerol carbonate and monoacetin from glycerol and carbon dioxide over $\mathrm{Cu}$ catalysts: The role of supports. J Chem Technol Biotechnol 90:1077-1085.

70. Li H, Jiao X, Li L, Zhao N, Xiao F, Wei W, Sun Y, Zhang B (2015) Synthesis of glycerol carbonate by direct carbonylation of glycerol with $\mathrm{CO}_{2}$ over solid catalysts derived from $\mathrm{Zn} / \mathrm{Al} / \mathrm{La}$ and $\mathrm{Zn} / \mathrm{Al} / \mathrm{La} / \mathrm{M}(\mathrm{M}=\mathrm{Li}, \mathrm{Mg}$ and $\mathrm{Zr}$ ) hydrotalcites. Catal Sci Technol 5:989_1005.

71. Liu J, Li Y, Zhang J, He D (2016) Glycerol carbonylation with $\mathrm{CO}_{2}$ to glycerol carbonate over $\mathrm{CeO}_{2}$ catalyst and the influence of $\mathrm{CeO}_{2}$ preparation methods and reaction parameters. Appl Catal A Gen 513:9-18.

72. Whiteoak CJ, Kielland N, Laserna V, Escudero-Adan EC, Martin E, Kleij AW (2013) A Powerful Aluminium Catalyst for the Synthesis of Highly Functional Organic 
Carbonates. J Am Chem Soc 135:1228-1231.

73. Gregory GL, Ulmann M, Buchard A (2015) Synthesis of 6-membered cyclic carbonates from 1,3-diols and low $\mathrm{CO}_{2}$ pressure: a novel mild strategy to replace phosgene reagents. RSC Adv 5:39404-39408.

74. Inoue Y, Itoh Y, Yen IF, Imaizumi S (1990) Palladium(0)-catalyzed carboxylative cyclized coupling of propargylic alcohol with aryl halides. J Mol Catal 60:L1-L3.

75. Kim H-S, Kim J-W, Kwon S-C, Shim S-C, Kim T-J (1997) Catalytic formation of carbamates and cyclic carbonates by copper complex of 2,5,19,22tetraaza[6,6](1,1')ferrocenophane-1,5-diene X-ray crystal structure of $[\mathrm{Cu}(1)] \mathrm{PF}_{6}$. $\mathrm{J}$ Organomet Chem 545-546:337-344.

76. Gu Y, Shi F, Deng Y (2004) Ionic Liquid as an Efficient Promoting Medium for Fixation of $\mathrm{CO}_{2}$ : Clean Synthesis of $\alpha$-Methylene Cyclic Carbonates from $\mathrm{CO}_{2}$ and Propargyl Alcohols Catalyzed by Metal Salts under Mild Conditions. J Org Chem 69:391-394.

77. Kimura T, Kamata K, Mizuno N (2012) A bifunctional tungstate catalyst for chemical fixation of $\mathrm{CO}_{2}$ at atmospheric pressure. Angew Chem Int Ed 51:6700-6703.

78. Song Q-W, Yu B, Li X-D, Ma R, Diao Z-F, Li R-G, Li W, He L-N (2014) Efficient chemical fixation of $\mathrm{CO}_{2}$ promoted by a bifunctional $\mathrm{Ag}_{2} \mathrm{WO}_{4} / \mathrm{Ph}_{3} \mathrm{P}$ system. Green Chem 16:1633-1638.

79. Yamada W, Sugawara Y, Hau MC, Ikeno T, Yamada T (2007) Silver-catalyzed incorporation of carbon dioxide into propargylic alcohols. Eur J Org Chem 2604-2607.

80. Yang Z-Z, Zhao Y, Zhang H, Yu B, Ma Z, Ji G, Liu Z (2014) Fluorinated microporous organic polymers: design and applications in $\mathrm{CO}_{2}$ adsorption and conversion. Chem Commun 50:13910-13913.

81. Jiang HF, Wang AZ, Liu HL, Qi CR (2008) Reusable polymer-supported amine-copper catalyst for the formation of $\alpha$-alkylidene cyclic carbonates in supercritical carbon dioxide. Eur J Org Chem 2309-2312.

82. Tang X, Qi C, He H, Jiang H, Ren Y, Yuan G (2013) Polystyrene-supported Nheterocyclic carbene-silver complexes as robust and efficient catalysts for the reaction of carbon dioxide and propargylic alcohols. Adv Synth Catal 355:2019-2028. 
83. Yang Z, Yu B, Zhang H, Zhao Y, Chen Y, Ma Z, Ji G, Gao X, Han B, Liu Z (2016) Metalated Mesoporous Poly(triphenylphosphine) with Azo Functionality: Efficient Catalysts for $\mathrm{CO}_{2}$ Conversion. ACS Catal 6:1268-1273.

84. Song QW, Chen WQ, Ma R, Yu A, Li QY, Chang Y, He LN (2015) Bifunctional silver(I) complex-catalyzed $\mathrm{CO}_{2}$ conversion at ambient conditions: Synthesis of $\alpha$-methylene cyclic carbonates and derivatives. ChemSusChem 8:821-827.

85. Cui M, Qian Q, He Z, Ma J, Kang X, Hu J, Liu Z, Han B (2015) Synthesizing Ag Nanoparticles of Small Size on a Hierarchical Porosity Support for the Carboxylative Cyclization of Propargyl Alcohols with $\mathrm{CO}_{2}$ under Ambient Conditions. Chem Eur J 21:15924-15928.

86. Qiu J, Zhao Y, Wang H, Cui G, Wang J (2016) AgX@ carbon (X = Br and I) as robust and efficient catalysts for the reaction of propargylic alcohols and $\mathrm{CO}_{2}$ to carbonates under ambient conditions. RSC Adv 6:54020-54026.

87. Hu J, Ma J, Zhu Q, Qian Q, Han H, Mei Q, Han B (2016) Zinc(II)-catalyzed reactions of carbon dioxide and propargylic alcohols to carbonates at room temperature. Green Chem 18:382-385.

88. Kayaki Y, Yamamoto M, Ikariya T (2009) N-Heterocyclic Carbenes as Efficient Organocatalysts for $\mathrm{CO}_{2}$ Fixation Reactions. Angew Chem Int Ed 48:4194-4197.

89. Wang Y-B, Wang Y-M, Zhang WZ, Lu X-B (2013) Fast $\mathrm{CO}_{2}$ sequestration, activation, and catalytic transformation using N-heterocyclic olefins. J Am Chem Soc 135:1199612003.

90. Wang Y-B, Sun D-S, Zhou H, Zhang W-Z, Lu X-B (2014) Alkoxide-functionalized imidazolium betaines for $\mathrm{CO}_{2}$ activation and catalytic transformation. Green Chem $16: 2266-2272$.

91. Minakata S, Sasaki I, Ide T (2010) Atmospheric $\mathrm{CO}_{2}$ Fixation by Unsaturated Alcohols Using tBuOI under Neutral Conditions. Angew Chem Int Ed 49:1309-1311.

92. Vara BA, Struble TJ, Wang W, Dobish MC, Johnston JN (2015) Enantioselective small molecule synthesis by carbon dioxide fixation using a dual Brønsted acid/base organocatalyst. J Am Chem Soc 137:7302-7305. 
1 93. Wang JL, He LN, Dou XY, Wu F (2009) Poly(ethylene glycol): An alternative solvent for the synthesis of cyclic carbonate from vicinal halohydrin and carbon dioxide. Aust $\mathbf{J}$ Chem 62:917-920.

94. Reithofer MR, Sum YN, Zhang Y (2013) Synthesis of cyclic carbonates with carbon dioxide and cesium carbonate. Green Chem 15:2086-2090.

95. Gregory GL, Ulmann M, Buchard A (2015) Synthesis of 6-membered cyclic carbonates from 1,3-diols and low $\mathrm{CO}_{2}$ pressure: a novel mild strategy to replace phosgene reagents. RSC Adv. 5, 39404-39408. 